\title{
Bisubstrate-type chemical probes identify GRP94 as a potential target of cytosine-containing adenosine analogs
}

Dany Pechalrieu ${ }^{\mathrm{a}}$, Fanny Assemat ${ }^{\mathrm{a}}$, Ludovic Halbya, ${ }^{\mathrm{a}}$, Marlene Marcellin ${ }^{\mathrm{c}}$, Pengrong Yan ${ }^{\mathrm{d}}$, Karima Chaoui ${ }^{\mathrm{c}}$, Sahil Sharma $^{\mathrm{d}}$, Gabriela Chiosis ${ }^{\mathrm{d}}$, Odile Burlet-Schiltz ${ }^{\mathrm{c}}$, Paola B. Arimondo ${ }^{\mathrm{a}, \mathrm{b} *}$ and Marie Lopez ${ }^{\mathrm{a}, \mathrm{e} *}$

\section{Supporting Information}

- Synthesis and characterization of compounds 6-17.

- ${ }^{1} \mathrm{H}$ and ${ }^{13} \mathrm{C}$ NMR spectra of compounds 1-5.

- Inhibition percentages of various DNMT, KHMTs and PRMTs for compounds 1-4.

- Table of proteomic analysis result tables (Table SI-1 to SI-5) and volcano plot (Graph SI-1 to SI-5) 


\section{General procedure for synthesis of compound 6,7 and 8}

To a suspension of ethyl cytosine-5-carboxylate [6, 7] (200 mg; $1.09 \mathrm{mmol})$ and $\mathrm{K}_{2} \mathrm{CO}_{3}(301 \mathrm{mg} ; 2.18$ mmol) in DMF ( $2 \mathrm{ml})$ was added the desired halide derivative (MOMCl $(91 \mu \mathrm{l} ; 1.20 \mathrm{mmol})$; BOMCl (tech. $70 \%)(167 \mu \mathrm{l} ; 1.20 \mathrm{mmol})$ or 2-(Boc-amino)ethylbromide (270mg; $1.20 \mathrm{mmol})$ ). The mixture was stirred overnight at room temperature. The mixture was filtrated, the solvent was removed and the residue was purified by silica gel flash chromatography using a linear gradient of ammonia $1 \mathrm{~N}$ in methanol $\left(0 \rightarrow 10 \% \mathrm{MeOH} / \mathrm{NH}_{3}\right)$ in $\mathrm{DCM}$ to give the desired product.

\section{N1-(methoxymethyl)-ethyl cytosine-5-carboxylate (6)}

Compound 6 was obtained as a white amorphous solid (191 mg; $0.90 \mathrm{mmol} ; 83 \%$ ).<smiles>CCOC(=O)c1cn(COC(=O)Cl)c(=O)nc1N</smiles>

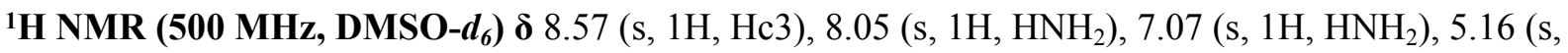
2H, Hc6), 4.27 (q, J=7.1Hz, 2H, HEt), 3.28 (s, 3H, Hc7), 1.30 (t, $J=7.1 \mathrm{~Hz}, 3 \mathrm{H}, \mathrm{HEt}$ ).

${ }^{13}$ C NMR (125 MHz, DMSO-d d $_{\mathbf{6}} \boldsymbol{\delta} 164.9$ (Cc1), 163.8 (Cc5), 154.30 (Cc4), 153.2 (Cc3), 95.5 (Cc2), 79.4 (Cc6), 61.1 (CEt), 56.7 (Cc7), 14.5 (CEt).

HRMS-ESI (m/z) calculated for $\mathrm{C}_{9} \mathrm{H}_{13} \mathrm{~N}_{3} \mathrm{Na}_{1} \mathrm{O}_{4}[\mathrm{M}+\mathrm{Na}]^{+}: 250.2092$; found: 250.2079 .

\section{N1-(benzoxymethyl)-ethyl cytosine-5-carboxylate (7)}

Compound 7 was obtained as a white amorphous solid (224 mg; $0.74 \mathrm{mmol} ; 68 \%$ ).<smiles></smiles>

${ }^{1}$ H NMR (500 MHz, DMSO-d $\left.\boldsymbol{d}_{\mathbf{6}}\right) \boldsymbol{\delta} 8.67$ (s, 1H, Hc3), 8.00 (s, 1H, HNH $)$, 7.77 (s, 1H, $\mathrm{HNH}_{2}$ ), 7.417.29 (m, 5H, Hc9 to Hc11), 5.28 (s, 2H, Hc6), 4.61 (s, 2H, Hc7), 4.27 (q, J=7.0Hz, 2H, HEt), 1.32 (t, $J=7.0 \mathrm{~Hz}, 3 \mathrm{H}, \mathrm{HEt})$.

${ }^{13}$ C NMR (125 MHz, DMSO-d $\boldsymbol{d}_{\mathbf{6}}$ ) $\boldsymbol{\delta} 165.9$ (Cc1), 164.0 (Cc5), 154.3 (Cc4), 153.0 (Cc3), 138.4 (Cc8), 128.7 (Cc10), 128.1 (Cc11), 127.9 (Cc9), 95.5 (Cc2), 78.4 (Cc6), 71.0 (Cc7), 61.0 (CEt), 14.9 (CEt).

HRMS-ESI (m/z) calculated for $\mathrm{C}_{15} \mathrm{H}_{17} \mathrm{~N}_{3} \mathrm{Na}_{1} \mathrm{O}_{4}[\mathrm{M}+\mathrm{Na}]^{+}:$326.1111; found: 326.1104 .

\section{N1-(2-((tert-butoxycarbonyl)amino)ethyl)-ethyl cytosine-5-carboxylate (8)}

Compound 8 was obtained as a white amorphous solid (218 mg; $0.67 \mathrm{mmol} ; 61 \%$ ). 
<smiles></smiles>

${ }^{1}$ H NMR (500 MHz, DMSO- $\boldsymbol{d}_{\mathbf{6}}$ ) $\boldsymbol{\delta} 8.29$ (s, 1H, Hc3), 7.83 (d, $\left.J=3.7 \mathrm{~Hz}, 1 \mathrm{H}, \mathrm{HNH}\right), 7.57$ (d, $J=3.6$ $\mathrm{Hz}, 1 \mathrm{H}, \mathrm{HNH}), 6.95(\mathrm{t}, J=6.0 \mathrm{~Hz}, 1 \mathrm{H}, \mathrm{HNH}), 4.24(\mathrm{q}, J=7.1 \mathrm{~Hz}, 2 \mathrm{H}, \mathrm{HEt}), 3.80$ (t, $J=5.5 \mathrm{~Hz}, 2 \mathrm{H}$, Hc6), 3.18 (q, $J=5.5 \mathrm{~Hz}, 2 \mathrm{H}, \mathrm{Hc} 7$ ), 1.32 (s, 9H, HBoc), 1.29 (t, $J=7.1 \mathrm{~Hz}, 3 \mathrm{H}, \mathrm{HEt}$ ).

${ }^{13}$ C NMR (125 MHz, DMSO-d $\boldsymbol{d}_{\mathbf{6}}$ ) $\boldsymbol{\delta} 165.1$ (Cc1), 163.8 (Cc5), 156.1 (CBoc), 154.3 (Cc4), 153.7 (Cc3), 94.2 (Cc2), 78.2 (CBoc), 60.8 (CEt), 50.4 (Cc6), 38.7 (Cc7), 28.5 (CBoc), 14.7 (CEt).

HRMS-ESI (m/z) calculated for $\mathrm{C}_{14} \mathrm{H}_{23} \mathrm{~N}_{4} \mathrm{O}_{5}[\mathrm{M}+\mathrm{H}]^{+}: 327.1681$; found: 327.1663 .

\section{General procedure for synthesis of compound 9, 10 and 11}

Compound 6 (200 mg; $0.88 \mathrm{mmol}$ ), compound 7 (200 mg; $0.62 \mathrm{mmol}$ ) or compound 8 (200 mg; 0.61 mmol) was solubilised in a solution of $\mathrm{NaOH} 0.25 \mathrm{M}$ in $\mathrm{MeOH} /$ water 1:1. The mixture was stirred overnight at room temperature and neutralised with concentrated $\mathrm{HCl}$. The solvents were evaporated under vacuum and $500 \mu 1$ of water was added to the mixture. After filtration the precipitate was wash with a minimum of $\mathrm{MeOH}$ and dried to afford the desired product.

\section{N1-(methoxymethyl)-5-carboxycytosine (9)}

Compound 9 was obtained as a white amorphous solid (147 mg; $0.74 \mathrm{mmol} ; 84 \%$ ).<smiles>Nc1nc(=O)n(COC(Cl)Cl)cc1C(=O)O</smiles>

${ }^{1}$ H NMR (500 MHz, DMSO-d $\left.\boldsymbol{d}_{\mathbf{6}}\right) \boldsymbol{\delta} 9.24$ (s, 1H, HNH2), 9.13 (s, 1H, HNH2), 8.84 (s, 1H, Hc3), 5.23 (s, 2H, Hc6), 3.33 (s, 3H, Hc7).

${ }^{13}$ C NMR (125 MHz, $\mathbf{C D C l}_{3}$ ) $\boldsymbol{\delta} 165.1$ (Cc5), 160.2 (Cc1), 154.9 (Cc3), 148.4 (Cc4), 96.5 (Cc2), 79.9 (Cc6), 57.7 (Cc7).

HRMS-ESI (m/z) calculated for $\mathrm{C}_{7} \mathrm{H}_{9} \mathrm{~N}_{3} \mathrm{Na}_{1} \mathrm{O}_{4}[\mathrm{M}+\mathrm{Na}]^{+}:$222.1552; found: 222.1550 .

\section{N1-(benzoxymethyl)-5-carboxycytosine (10)}

Compound 10 was obtained as a white amorphous solid (159 mg; $0.54 \mathrm{mmol} ; 87 \%$ ).

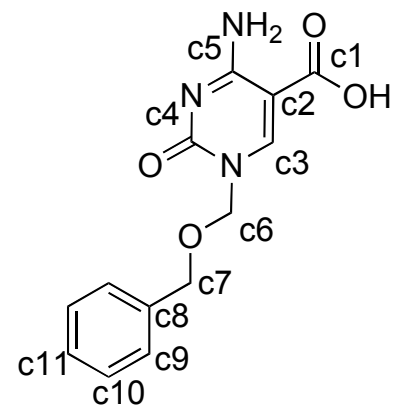


${ }^{1}$ H NMR (500 MH, DMSO-d $\left.\boldsymbol{d}_{\boldsymbol{6}}\right) \boldsymbol{\delta} 13.7$ (brs, 1H, HCOOH), 9.57 (s, 1H, Hc3), 8.03 (s, 1H, HNH2), 7.92 (s, 1H, HNH2), 7.38-7.27 (m, 5H, Hc9 to c11), 5.30 (s, 2H, Hc6), 4.60 (s, 2H, Hc7).

${ }^{13}$ C NMR (125 MHz, DMSO-d 6 ) $\delta 166.7$ (Cc5), 164.1 (Cc1), 154.3 (Cc4), 153.3 (Cc3), 138.0 (Cc8), 128.7 (Cc10), 128.1 (Cc11), 128.0 (Cc9), 96.0 (Cc2), 78.2 (Cc6), 71.0 (Cc7).

HRMS-ESI (m/z) calculated for $\mathrm{C}_{13} \mathrm{H}_{13} \mathrm{~N}_{3} \mathrm{Na}_{1} \mathrm{O}_{4}[\mathrm{M}+\mathrm{Na}]^{+}:$298.0798; found: 298.0789.

\section{N1-(2-((tert-butoxycarbonyl)amino)ethyl)-5-carbethoxycytosine (11)}

Compound 11 was obtained as a white amorphous solid (159 mg; $0.47 \mathrm{mmol} ; 76 \%$ ).<smiles>CC(C)(C)OC(=O)NCCn1cc(C(=O)O)c(N)nc1=O</smiles>

${ }^{1}$ H NMR (500 MHz, DMSO-d d $_{\text {) }} \boldsymbol{\delta} 13.0$ (s, 1H, HCOOH), 8.29 (s, 1H, Hc3), 7.82 (s, 2H, HNH), 6.94 (t, $J=6.0 \mathrm{~Hz}, 1 \mathrm{H}, \mathrm{HNH}), 3.81$ (t, $J=5.3 \mathrm{~Hz}, 2 \mathrm{H}, \mathrm{Hc} 6), 3.2$ (q, $J=5.6 \mathrm{~Hz}, 2 \mathrm{H}, \mathrm{Hc} 7), 1.3(\mathrm{~s}, 8 \mathrm{H}$, HBoc).

${ }^{13}$ C NMR (125 MHz, DMSO-d $\left.\boldsymbol{d}_{6}\right) \boldsymbol{\delta} 167.1$ (Cc1), 164.2 (Cc5), 156.1 (CBoc), 154.5 (Cc4), 153.8 (Cc3), 94.7 (Cc2), 78.2 (CBoc), 50.1 (Cc6), 38.8 (Cc7), 28.5 (CBoc).

HRMS-ESI (m/z) calculated for $\mathrm{C}_{12} \mathrm{H}_{18} \mathrm{~N}_{4} \mathrm{NaO}_{5}[\mathrm{M}+\mathrm{Na}]^{+}: 321.1169$; found: 321.1158 .

\section{General procedure for synthesis of compounds 12, 13 and 14}

To a suspension of compound $\mathbf{9 , 1 0}$ or $11(0.41 \mathrm{mmol})$ and HATU (312 $\mathrm{mg}$; $0.82 \mathrm{mmol})$ in DMF was added DiPEA $(214 \mu \mathrm{l} ; 1.26 \mathrm{mmol})$. The mixture was stirred at room temperature for $5 \mathrm{~min}$ then 2',3'$O$-isopropylidene-5'-deoxy-5'-aminomethyldenosine [8] was added to the mixture that was stirred again for $2 \mathrm{~h}$. The solvent was removed and the residue was purified by silica gel flash chromatography using a linear gradient of ammonia $1 \mathrm{~N}$ in methanol $\left(0 \rightarrow 15 \% \mathrm{MeOH} / \mathrm{NH}_{3}\right)$ in $\mathrm{DCM}$ to give the desired product.

\section{1-(methoxymethyl)- $N$-(2',3'-O-isopropylidene-(5'-deoxyadenosin-5'-yl)methyl)cytosine-5-} carboxamide (12)

Compound 12 was obtained as a white amorphous solid (171 mg; $0.34 \mathrm{mmol} ; 83 \%$ ).

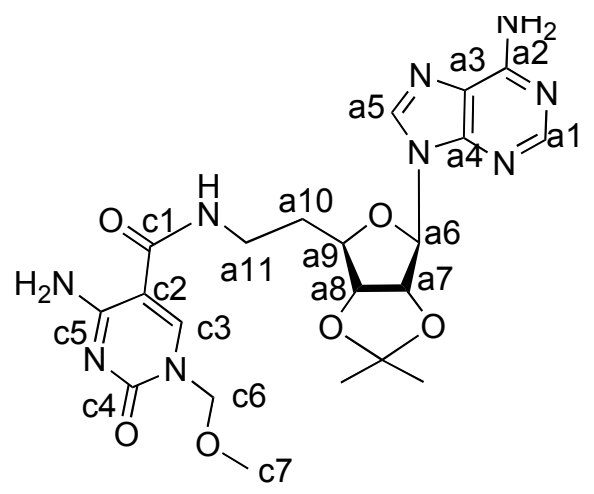

${ }^{1}$ H NMR (500 MHz, DMSO-d $\boldsymbol{d}_{\boldsymbol{6}}$ ) $\boldsymbol{\delta}$ 8.38-8.31 (m, 3H, Ha5 and Hc3 and HNH), 8.27 (brs, 1H, HNH), 8.17 (s, 1H, Ha1), 7.78 (s 1H, HNH), 7.35 (brs, 2H, HNH), 6.13 (d, J=2.9Hz, 1H, Ha6), 5.49 (dd, 
$J=2.6,6.3 \mathrm{~Hz}, 1 \mathrm{H}, \mathrm{Ha} 7), 5.03$ (s, 1H, Hc6), 4.91 (dd, $J=3.5,6.2 \mathrm{~Hz}, 1 \mathrm{H}, \mathrm{Ha} 8), 4.21-4.15$ (m, 1H, Ha9), 3.27 (s, 3H, Hc7), 3.23-3.13 (m, 2H, Ha11), 1.94-1.76 (m, 2H, Ha10), 1.53 (s, 3H, HiPr), 1.32 (s, 3H, HiPr).

${ }^{13}$ C NMR (125 MHz, DMSO-d $\boldsymbol{d}_{\text {) }} \boldsymbol{\delta} 165.4$ (Cc5), 164.5 (Cc1), 156.6 (Ca2), 154.5 (Cc4), 153.2 (Ca1), 149.4 (Ca4), 148.5 (Cc3), 140.4 (Ca5), 119.5 (Ca3), 113.9 (Cipr), 98.9 (Cc2), 88.9 (Ca6), 84.0 (Ca7), 83.9 (Ca8), 83.5 (Ca9), 79.6 (Cc6), 48.4 (Cc6), 56.7 (Cc7), 36.2 (Ca11), 33.2 (Ca10), 27.5 (CiPr), 25.7 (CiPr)

HRMS-ESI (m/z) calculated for $\mathrm{C}_{21} \mathrm{H}_{28} \mathrm{~N}_{9} \mathrm{O}_{6}[\mathrm{M}+\mathrm{H}]^{+}:$502.2157; found: 502.2163 .

\section{1-(benzoxymethyl)- $N$-(2',3'-O-isopropylidene-(5'-deoxyadenosin-5'-yl)methyl)cytosine-5- carboxamide (13)}

Compound 13 was obtained as a white amorphous solid (208 mg; $0.36 \mathrm{mmol} ; 88 \%$ ).

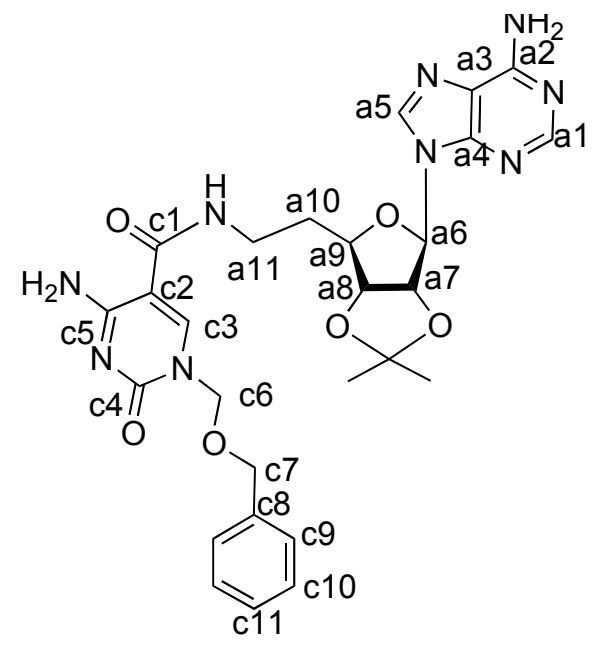

${ }^{1}$ H NMR (500 MHz, DMSO-d $\left.\boldsymbol{d}_{\mathbf{6}}\right) \boldsymbol{\delta} 8.39$ (s, 1H, Hc3) and 8.35 (s, 1H, Ha5), 8.32 (t, $1 \mathrm{H}, J=5.4 \mathrm{~Hz}$, $\mathrm{HNH}$ ), 8.28 (brs, 1H, HNH), 8.18 (s, 1H, Ha1), 7.80 (brs 1H, HNH), 7.38-7.26 (m, 7H, Hc9to10 and $2 \mathrm{HNH}$ ), 6.13 (d, $J=2.6 \mathrm{~Hz}, 1 \mathrm{H}, \mathrm{Ha}$ ), 5.50 (dd, $J=2.6,6.4 \mathrm{~Hz}, 1 \mathrm{H}, \mathrm{Ha} 7$ ), 5.19 (s, 1H, Hc6), 4.92 (dd, $J=3.5,6.4 \mathrm{~Hz}, 1 \mathrm{H}, \mathrm{Ha} 8), 4.59$ (s, 2H, Hc7), 4.21-4.15 (m, 1H, Ha9), 3.23-3.13 (m, 2H, Ha11), 1.951.77 (m, 2H, Ha10), 1.53 (s, 3H, HiPr), 1.32 (s, 3H, HiPr).

${ }^{13}$ C NMR (125 MHz, DMSO-d $\boldsymbol{d}_{6}$ ) $\boldsymbol{\delta} 165.4$ (Cc5), 164.5 (Cc1), 156.6 (Ca2), 154.5 (Cc4), 153.2 (Ca1), 149.4 (Ca4), 148.6 (Cc3), 140.4 (Ca5), 138.0 (Cc8), 128.7 (Cc10), 128.1 (Cc11), 128.0 (Cc9), 119.6 (Ca3), 113.9 (Cipr), 99.0 (Cc2), 88.9 (Ca6), 84.0 (Ca7), 83.9 (Ca8), 83.5 (Ca9), 78.3 (Cc6), 71.0 (Cc7), 48.4 (Cc6), 56.5 (Cc7), 36.1 (Ca11), 33.2 (Ca10), 27.5 (CiPr), 25.7 (CiPr)

HRMS-ESI (m/z) calculated for $\mathrm{C}_{27} \mathrm{H}_{32} \mathrm{~N}_{9} \mathrm{O}_{6}[\mathrm{M}+\mathrm{H}]^{+}:$578.2470; found: 578.2463.

1-(2-((tert-butoxycarbonyl)amino)ethyl)- $N$-(2',3'-O-isopropylidene-(5'-deoxyadenosin-5'yl)methyl)cytosine-5-carboxamide (14)

Compound 14 was obtained as a white amorphous solid (174 mg; $0.29 \mathrm{mmol} ; 71 \%$ ). 


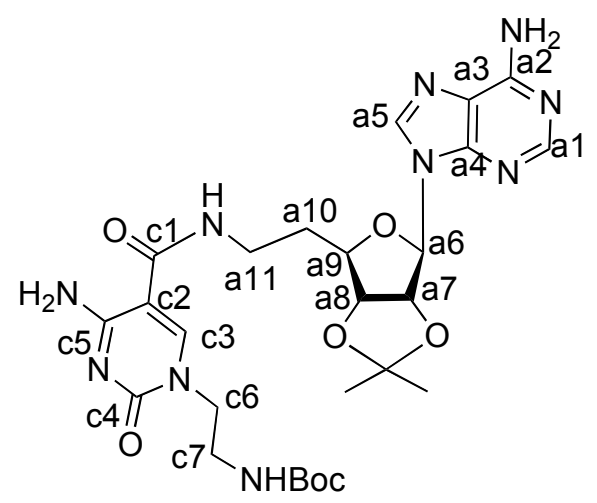

${ }^{1}$ H NMR (500 MHz, DMSO-d d $_{\mathbf{6}} \boldsymbol{\delta} 8.35$ (s, 1H, Hc3), 8.22 (t, $\left.J=5.4 \mathrm{~Hz}, 1 \mathrm{H}, \mathrm{HNH}\right), 8.17$ (s, 1H, Ha1), 8.15 (brs, 2H, Ha5 and HNH), 7.55 (brs, $1 \mathrm{H}, \mathrm{HNH}$ ), 7.35 (s, 2H, HNH), 6.94 (t, $J=5.8 \mathrm{~Hz}, 1 \mathrm{H}$, HNH), 6.13 (d, $J=2.6 \mathrm{~Hz}, 1 \mathrm{H}, \mathrm{Ha}$ ), 5.51 (dd, $J=6.3,2.7 \mathrm{~Hz}, 1 \mathrm{H}, \mathrm{Ha} 7), 4.92$ (dd, $J=6.3,3.5 \mathrm{~Hz}$, 1H, Ha8), 4.18 (ddd, $J=8.8,5.4,3.5 \mathrm{~Hz}, 1 \mathrm{H}, \mathrm{Ha} 9), 3.79-3.64$ (m, 2H, Hc6), 3.17 (m, 4H, Hc7 and Ha11), 1.97-1.74 (m, 2H, Ha10), 1.54 (s, 3H, HiPr), 1.33 (s, 3H, HiPr), 1.32 (s, 9H, HBoc),

${ }^{13}$ C NMR (125 MHz, DMSO-d d $_{\text {) }} \boldsymbol{\delta} 165.7$ (Cc5), 164.4 (Cc1), 156.6 (Ca2), 156.1 (CBoc), 154.5 (Cc4), 153.2 (Ca1), 149.4 (Ca4), 149.3 (Ca5), 140.3 (Cc3), 119.6 (Ca3), 113.9 (CiPr), 97.9 (Cc2), 88.9 (Ca6), 84.0 (Ca9), 83.9 (Ca8), 83.5 (Ca7), 78.3 (CBoc), 49.9 (Cc6), 49.0, 38.9 (Cc7), 36.1 (Ca11), 33.4 (Ca10), 28.6 (CBoc), 27.5 (CiPr), 25.7 (CiPr),

HRMS-ESI (m/z) calculated for $\mathrm{C}_{26} \mathrm{H}_{36} \mathrm{~N}_{10} \mathrm{O}_{7}[\mathrm{M}+\mathrm{H}]^{+}: 601.2841$; found: 601.2823 .

\section{N-(4-(4-aminobenzoyl)phenyl)hex-5-ynamide (15)}

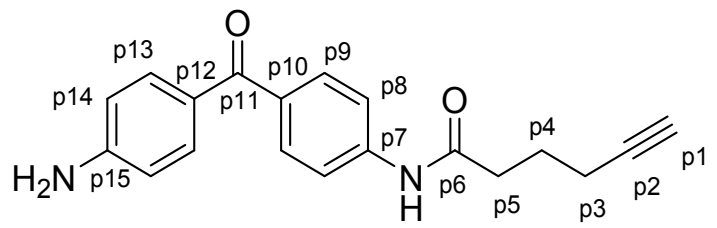

1-[Bis(dimethylamino)methylene]-1H-1,2,3-triazolo[4,5-b]pyridinium 3-oxid hexafluorophosphate (HATU) (2.05 g; $5.3 \mathrm{mmol})$ was solubilized in DMA (10 ml) at RT under argon. To the solution was added 5-hexynoic acid $(0.45 \mathrm{ml} ; 4.1 \mathrm{mmol})$ and $N, N$-diisopropylethylamine (DIPEA) $(2 \mathrm{ml} ; 11.5$ $\mathrm{mmol})$. Reaction mixture was stirred for $15 \mathrm{~min}$ before addition of 4,4'-diaminobenzophenone ( $1 \mathrm{~g}$; $4.9 \mathrm{mmol}$ ) and was then further stirred for $2 \mathrm{~h}$ at room temperature. The residue was diluted with ethyl acetate and washed with saturated $\mathrm{NaHCO}_{3}, 10 \%$ aqueous citric acid and dried over sodium sulfate. The solvent was removed and the residue was purified by silica gel flash chromatography using a linear gradient of methanol $(0 \rightarrow 20 \% \mathrm{MeOH})$ in dichloromethane to obtain the title compound $\mathbf{1 5}$ as a yellow solid (926 mg; $3.0 \mathrm{mmol} ; 73 \%$ ).

${ }^{1}$ H NMR (500 MHz, DMSO) $\delta 10.22(\mathrm{~s}, 1 \mathrm{H}, \mathrm{HNH}), 7.71$ (d, $\left.J=8.5 \mathrm{~Hz}, 2 \mathrm{H}, \mathrm{Hp} 8\right), 7.59$ (d, $J=8.6 \mathrm{~Hz}$, 2H, Hp9), 7.50 (d, $J=8.6 \mathrm{~Hz}, 2 \mathrm{H}, \mathrm{Hp} 13$ ), 6.59 (d, $J=8.6 \mathrm{~Hz}, 2 \mathrm{H}, \mathrm{Hp} 14), 6.09$ (s, 2H, HNH), 2.83 (t, $J=2.6 \mathrm{~Hz}, 1 \mathrm{H}, \mathrm{Hp} 1), 2.47$ (t, $J=7.4 \mathrm{~Hz}, 2 \mathrm{H}, \mathrm{Hp5}), 2.24$ (dt, $J=2.6,7.1 \mathrm{~Hz}, 2 \mathrm{H}, \mathrm{Hp} 3), 1.77$ (quint, $J=7.3 \mathrm{~Hz}, 2 \mathrm{H}, \mathrm{Hp} 4)$.

${ }^{13}$ C NMR (125 MHz, DMSO) $\delta 192.4$ (Cp11), 171 (Cp6), 153.4 (Cp15), 142 (Cp7), 133.2 (Cp10), 132.4 (Cp13), 130.2 (Cp9), 124.1 (Cp12), 118.1 (Cp8), 112.5 (Cp14), 83.9 (Cp2), 71.7 (Cp1), 35.2 (Cp5), 23.8 (Cp4), 17.3 (Cp3).

HRMS-ESI (m/z) calculated for $\mathrm{C}_{19} \mathrm{H}_{19} \mathrm{~N}_{2} \mathrm{O}_{2}[\mathrm{M}+\mathrm{H}]^{+}:$307.1368; found: 307.1382 . 


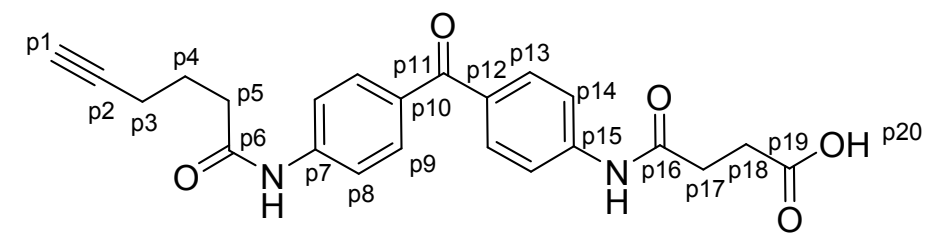

Succinic anhydride (100 $\mathrm{mg}, 1 \mathrm{mmol})$ was dissolved in $2 \mathrm{ml}$ of dioxane and $\mathrm{N}$-(4-(4aminobenzoyl)phenyl)hex-5-ynamide $(184 \mathrm{mg}, 0.6 \mathrm{mmol})$ in $2 \mathrm{ml}$ of dioxane was slowly added. The reaction mixture was heated to $80{ }^{\circ} \mathrm{C}$ for $4 \mathrm{~h}$. The solvent was then removed and the residue was purified by silica gel flash chromatography using a linear gradient of methanol $(0 \rightarrow 20 \% \mathrm{MeOH})$ in dichloromethane to obtain the title compound $\mathbf{1 6}$ as a yellow solid (207 $\mathrm{mg}$; $0.51 \mathrm{mmol} ; 85 \%$ ).

${ }^{1}$ H NMR (500 MHz, DMSO) $\delta 12.17$ (brs, 1H, Hp20), 10.34 (s, 1H, NH), 10.30 (s, 1H, NH), 7.787.73 (m, 4H, Hp9, Hp13), 7.72-7.67 (m, 4H, Hp8, Hp14), 2.83 (t, $J=2.5$ Hz, 1H, Hp1), 2.62 (t, $J=6.5$ $\mathrm{Hz}, 2 \mathrm{H}, \mathrm{Hp} 17), 2.54$ (t, $J=6.5 \mathrm{~Hz}, 2 \mathrm{H}, \mathrm{Hp} 18), 2.48$ (t, $J=7.1 \mathrm{~Hz}, 2 \mathrm{H}, \mathrm{Hp} 5), 2.24$ (dt, $J=2.8,6.9 \mathrm{~Hz}, 2 \mathrm{H}$, Hp3), 1.78 (quint, $J=7.4 \mathrm{~Hz}, 2 \mathrm{H}, \mathrm{Hp} 4$ ).

${ }^{13}$ C NMR (125 MHz, DMSO) $\delta 193.4$ (Cp11), 173.8 (Cp19), 171.2 (Cp6), 170.8 (Cp16), 143.1 (Cp15), 143 (Cp7), 131.7 (Cp10 and Cp12), 130.9 (Cp9 and Cp13), 118.1 (Cp8 and Cp14), 83.9 (Cp2), 71.8 (Cp1), 35.2 (Cp5), 31.2 (Cp17), 28.7 (Cp18), 23.8 (Cp4), 17.4 (Cp3).

HRMS-ESI (m/z) calculated for $\mathrm{C}_{23} \mathrm{H}_{23} \mathrm{~N}_{2} \mathrm{O}_{5}[\mathrm{M}+\mathrm{H}]^{+}: 407.1601$; found: 407.1605.

\section{5-(Boc-amino)pentanoic acid (17)}<smiles>O=C(O)CCCCCCCC(=O)O</smiles>

5-Aminopentanoic acid $(0.981 \mathrm{~g} ; 8.4 \mathrm{mmol})$ was solubilized in dioxane $(20 \mathrm{ml})$. A solution of $\mathrm{NaOH}$ $(0.34 \mathrm{~g} ; 8.5 \mathrm{mmol})$ in water $(10 \mathrm{ml})$ was added and the mixture was cooled down to $0{ }^{\circ} \mathrm{C}$. Di-tert-butyl dicarbonate $(2.4 \mathrm{~g} ; 11.0 \mathrm{mmol})$ was then added and the reaction mixture was stirred at room temperature for $16 \mathrm{~h}$. The solution was concentrated under reduced pressure. The basic residue was diluted in $\mathrm{H}_{2} \mathrm{O}$ and washed with EtOAc. The aqueous phase was acidified to $\mathrm{pH} 1-2$ with $1 \mathrm{~N} \mathrm{HCl}$, extracted with EtOAc and dried over sodium sulfate. The solvent was removed to afford the title compound 17 as a clear oil (1.58 g; $7.2 \mathrm{mmol} ; 87 \%)$.

${ }^{1} \mathbf{H}$ NMR (500 MHz, CDCl $)_{3} \boldsymbol{\delta} 4.59$ (s, 1H, NH), 3.15-3.04 (m, 2H, Hp5), 2.35 (t, J=7.3 Hz, 2H, Hp2), 1.64 (quint, $J=7.7 \mathrm{~Hz}, 2 \mathrm{H}, \mathrm{Hp} 3$ ), 1.51 (quint, $J=7.3 \mathrm{~Hz}, \mathrm{Hp} 4$ ), 1.41 (s, 9H, HBoc)

${ }^{13}$ C NMR (125 MHz, CDCl ${ }_{3}$ ) $\delta 178.9$ (Cp1), 156.3 (CBoc), 79.5 (CBoc), 40.3 (Cp5), 33.7 (Cp2), 29.6 (Cp4), 28.6 (CBoc), 22 (Cp3).

HRMS-ESI (m/z) calculated for $\mathrm{C}_{10} \mathrm{H}_{20} \mathrm{NO}_{4}[\mathrm{M}+\mathrm{H}]^{+}:$218.1387; found: 218.1392 . 
${ }^{1} \mathrm{H}$ and ${ }^{13} \mathrm{C}$ NMR of compound 1

$$
\text { (1) }
$$

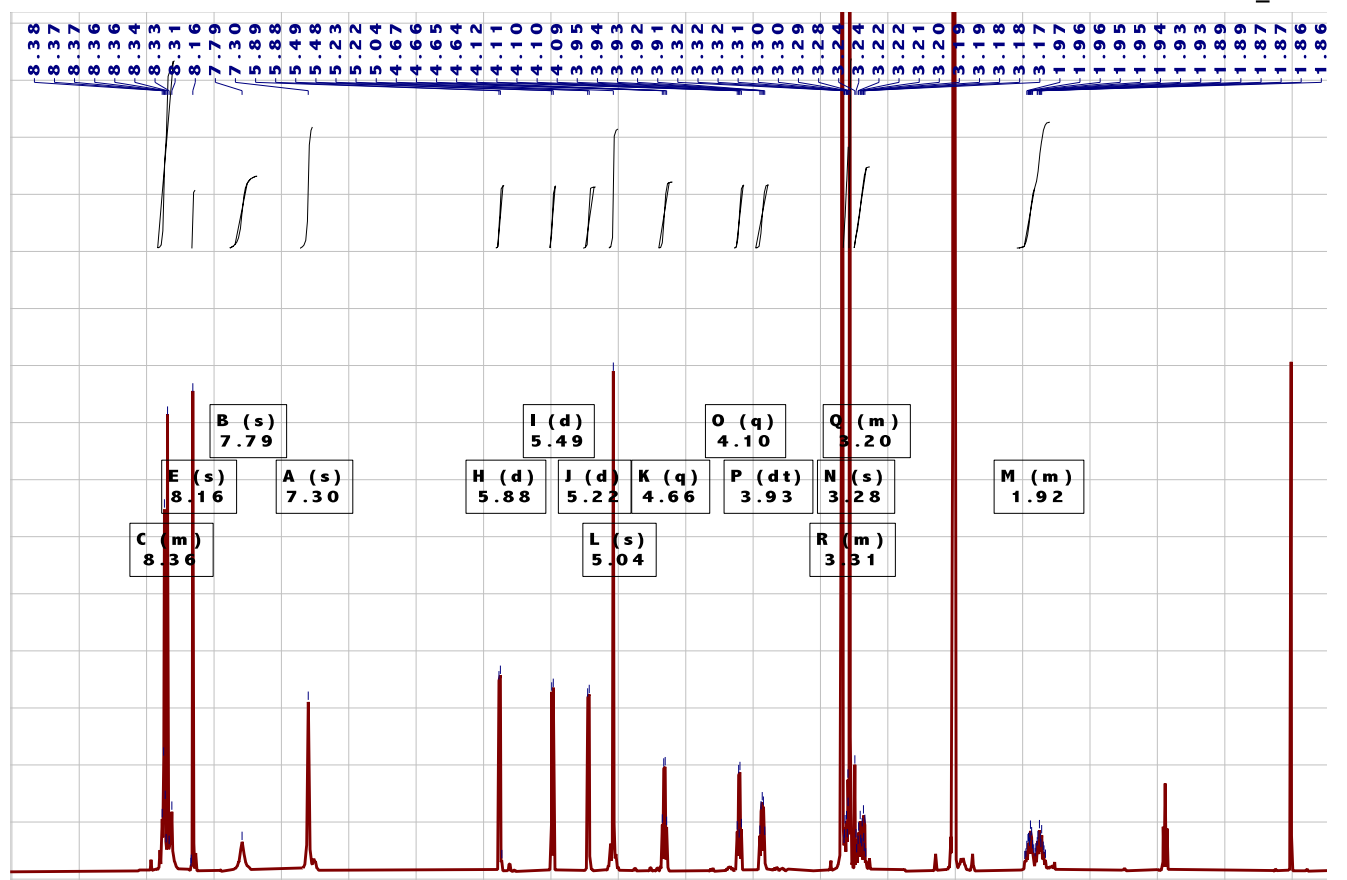

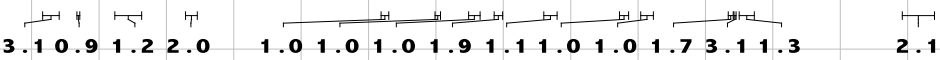

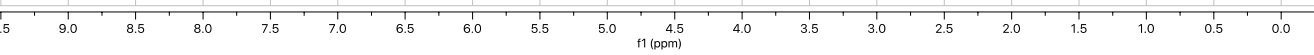

min.

in

i)

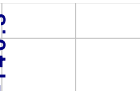

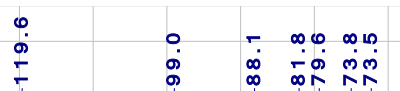

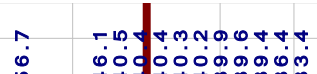

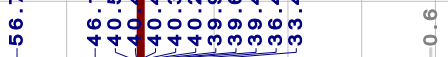

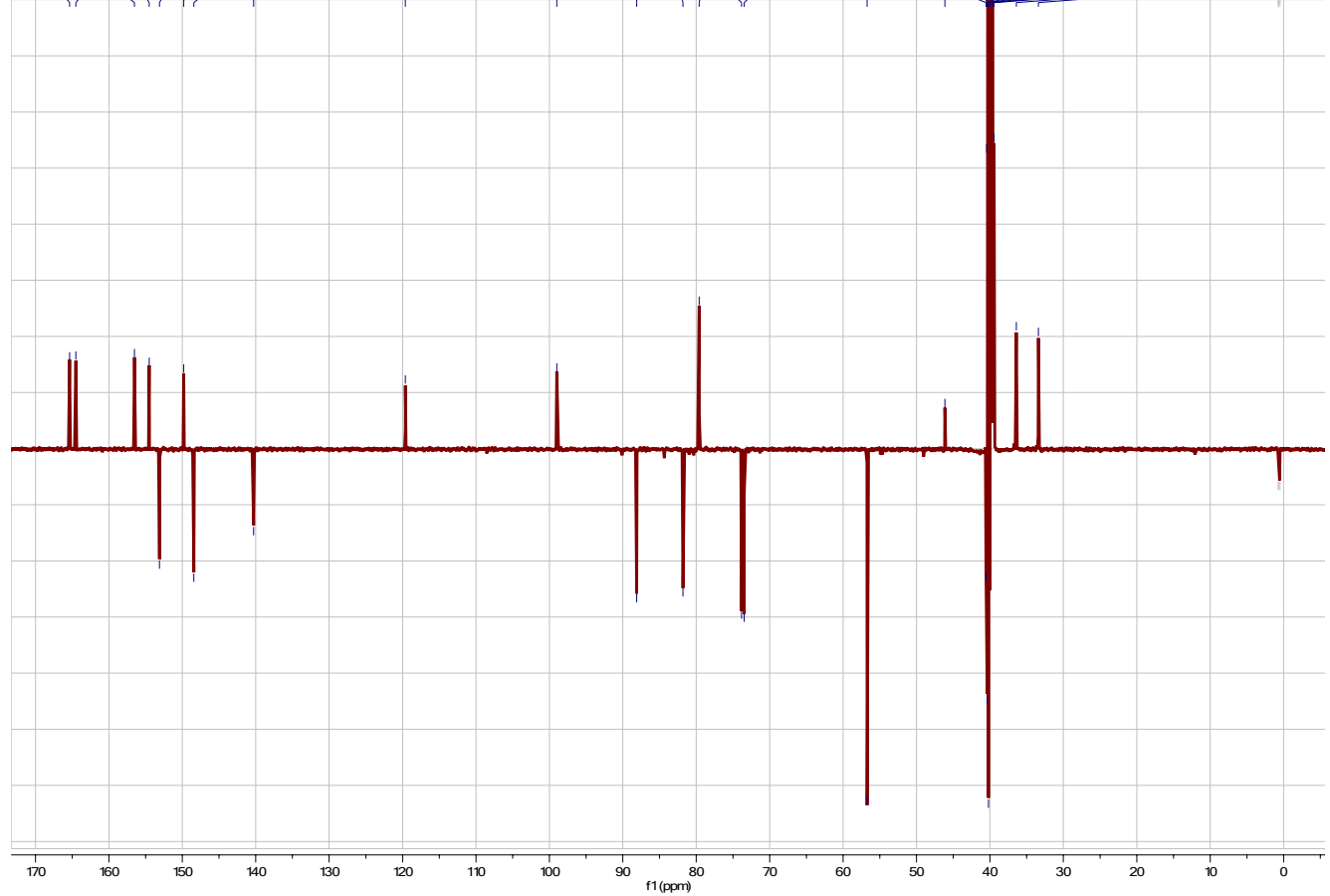


${ }^{1} \mathrm{H}$ and ${ }^{13} \mathrm{C}$ NMR of compound 2
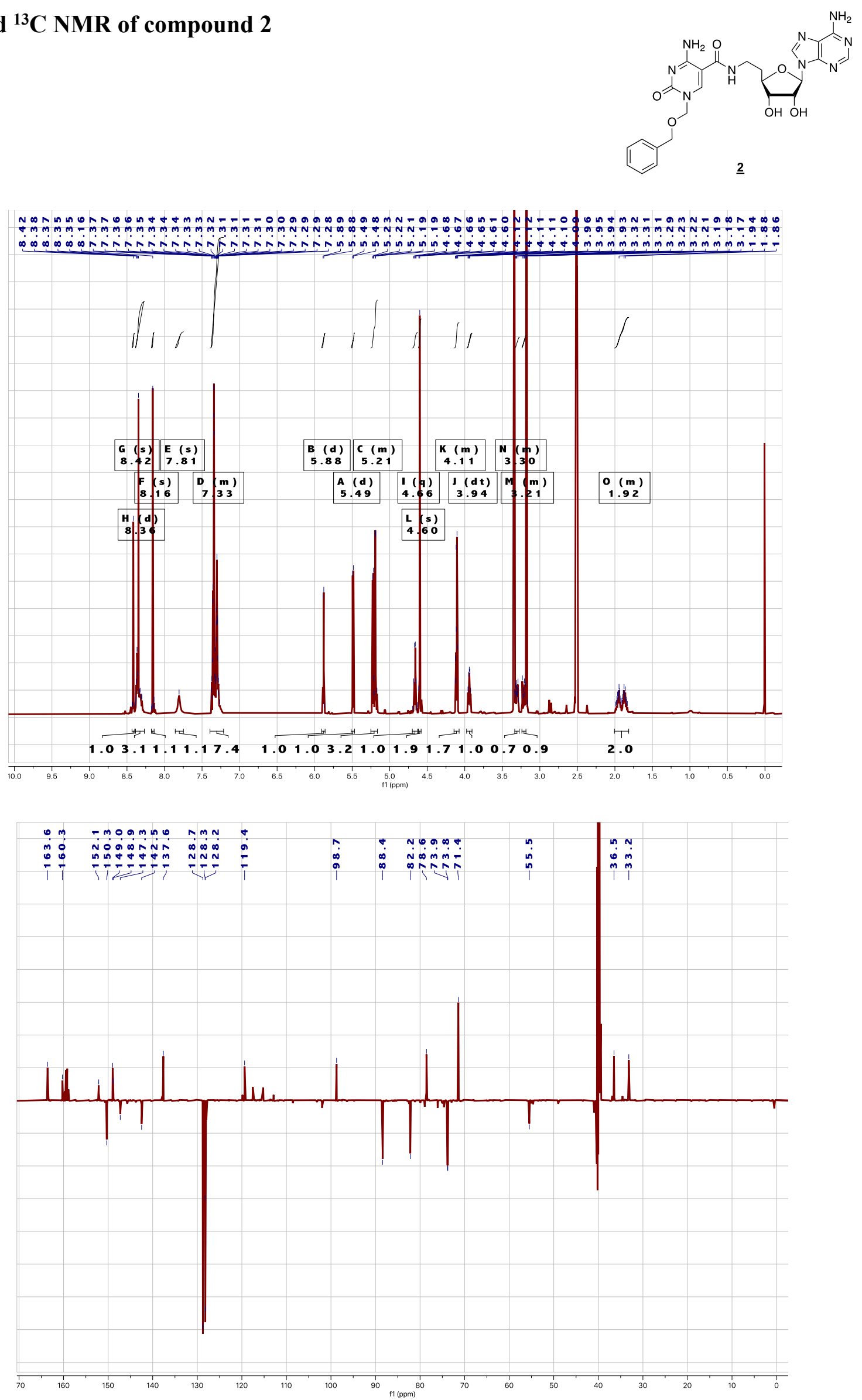
${ }^{1} \mathrm{H}$ and ${ }^{13} \mathrm{C}$ NMR of compound 3
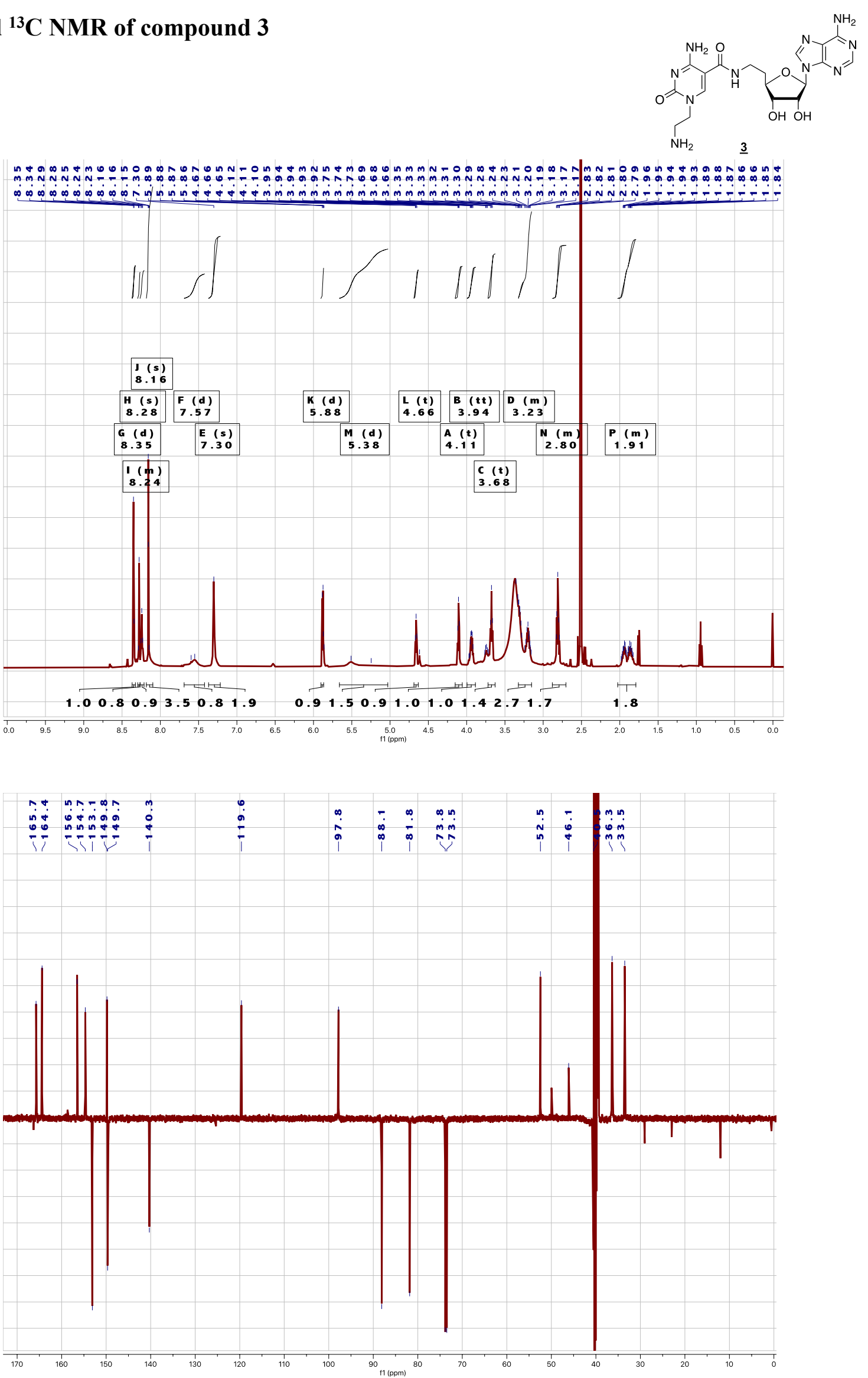
${ }^{1} \mathrm{H}$ and ${ }^{13} \mathrm{C}$ NMR of compound 4

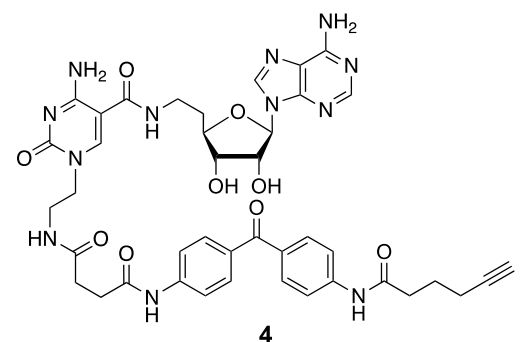

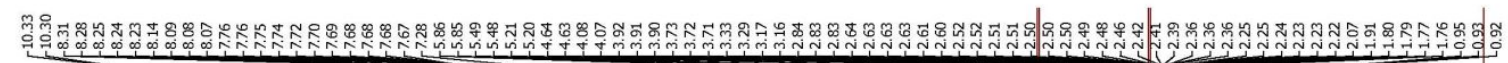

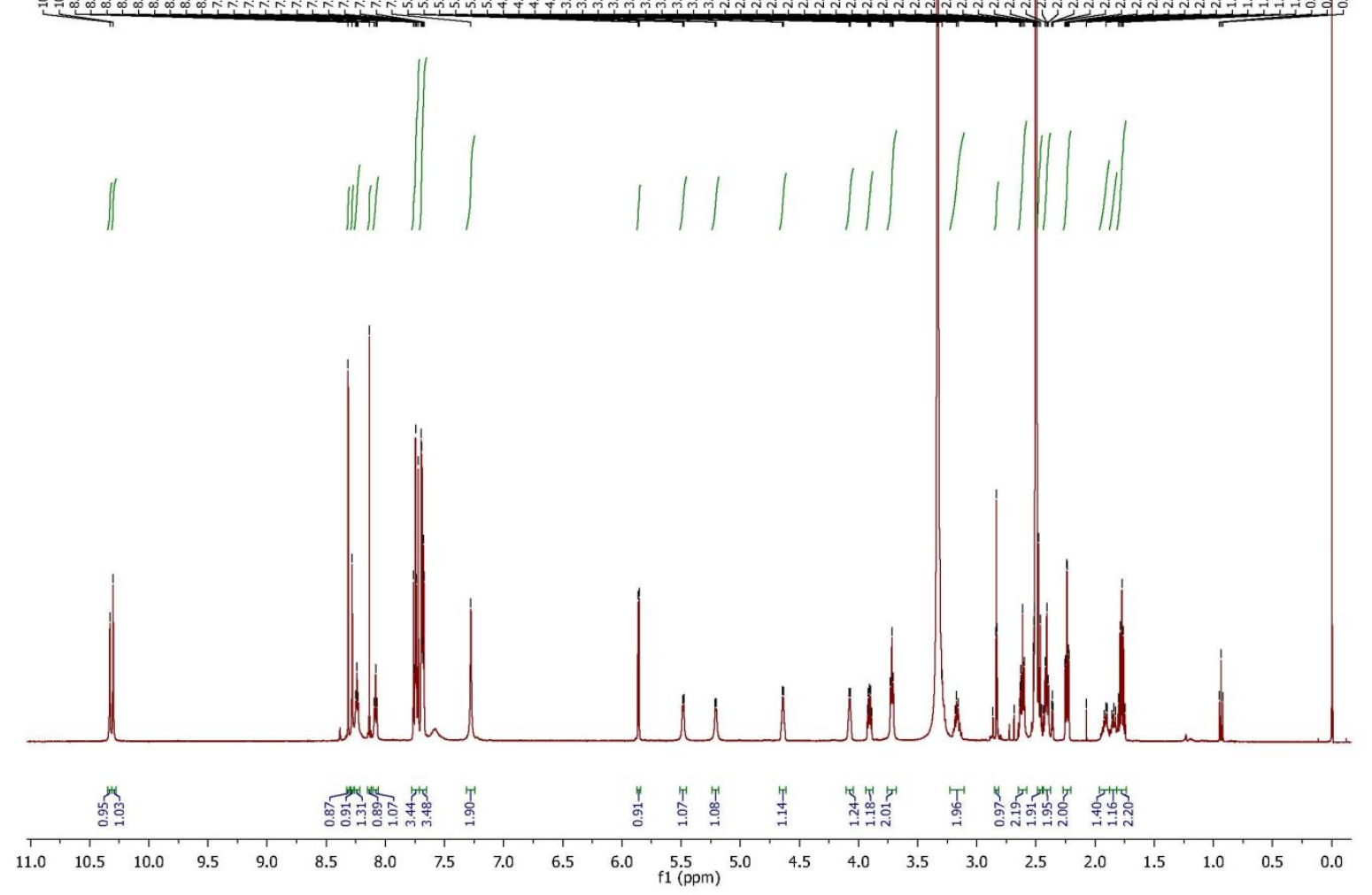

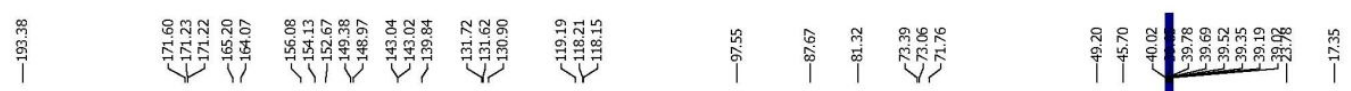

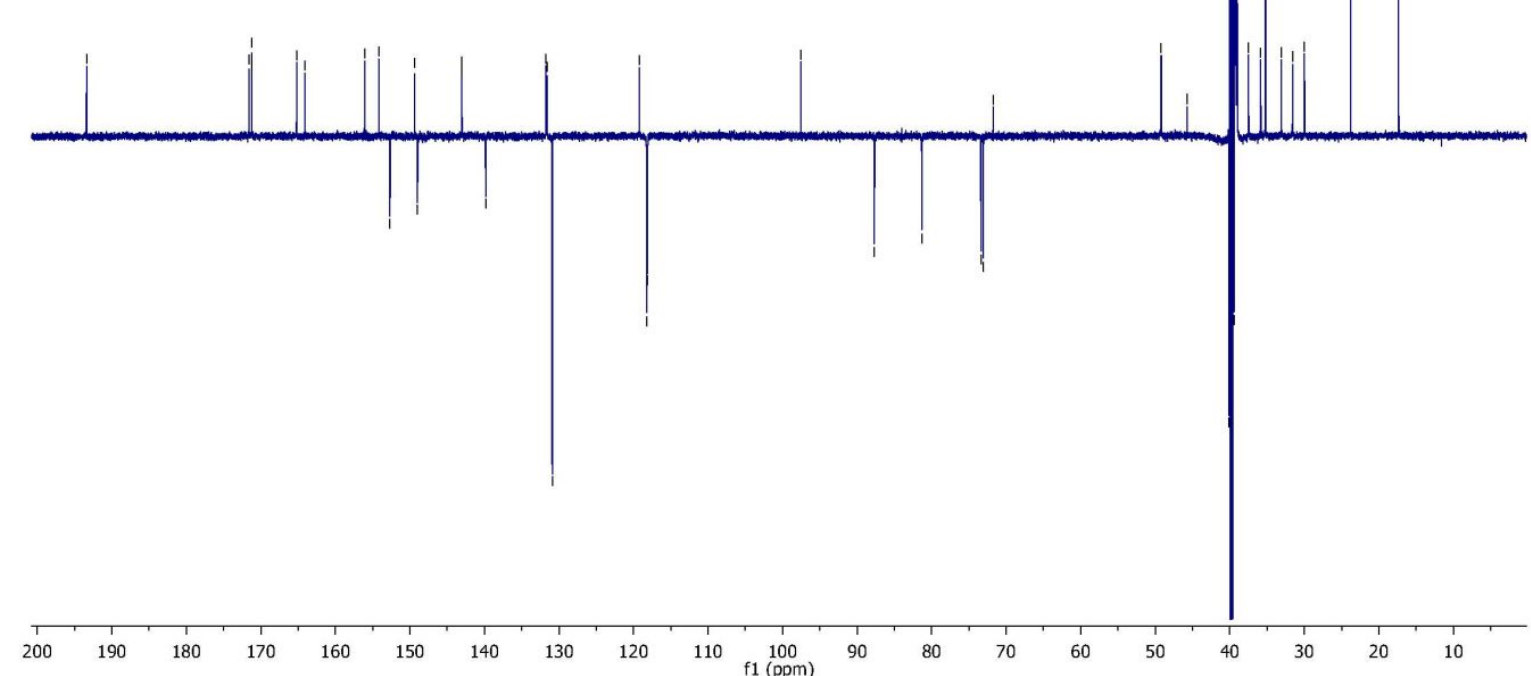


${ }^{1} \mathrm{H}$ and ${ }^{13} \mathrm{C}$ NMR of compound 5
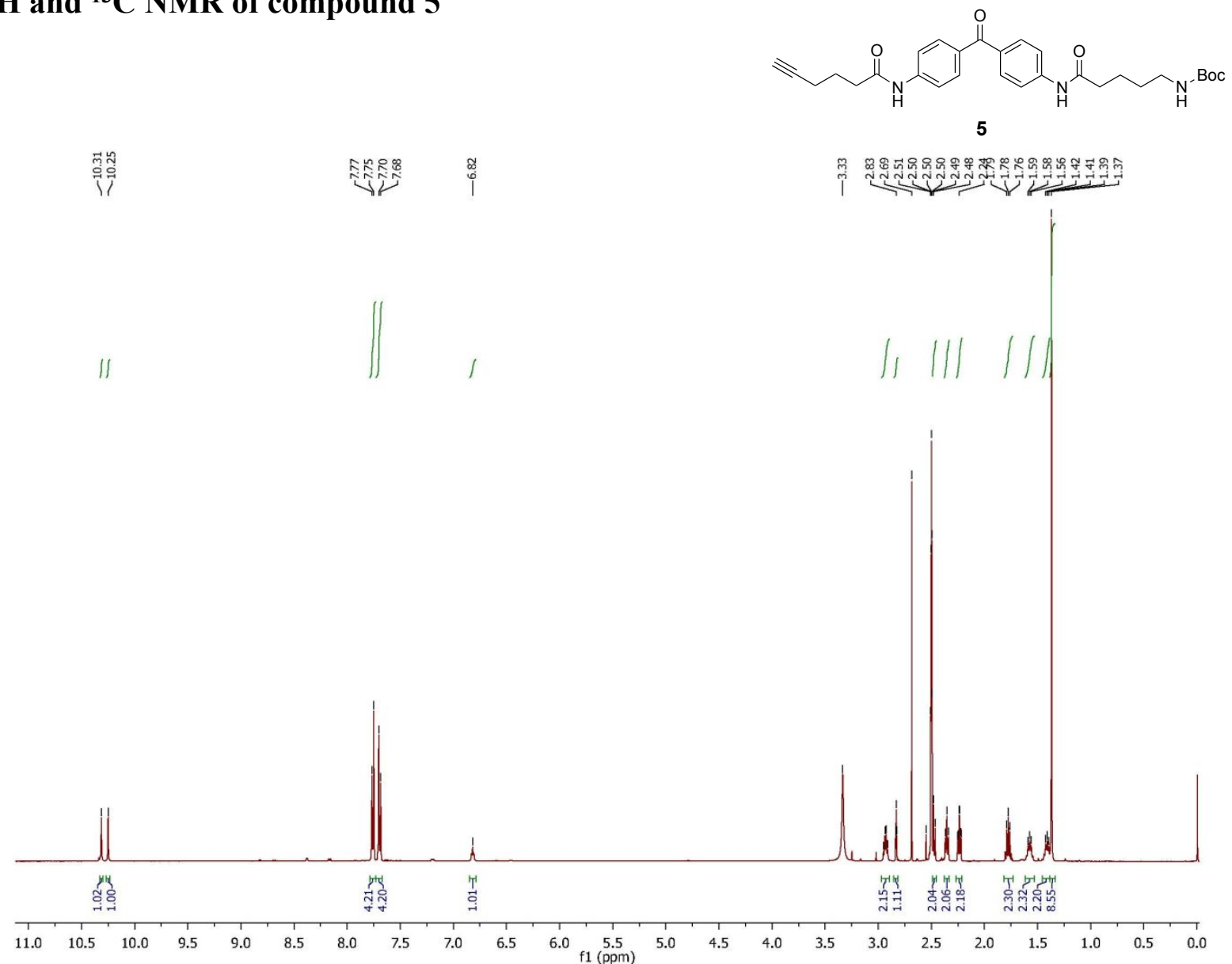

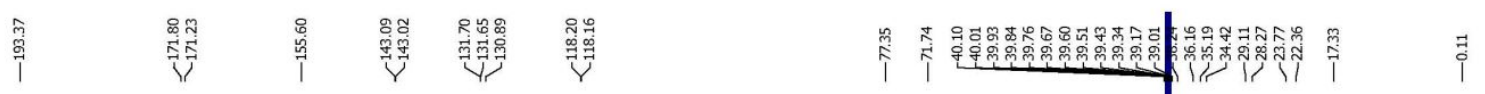

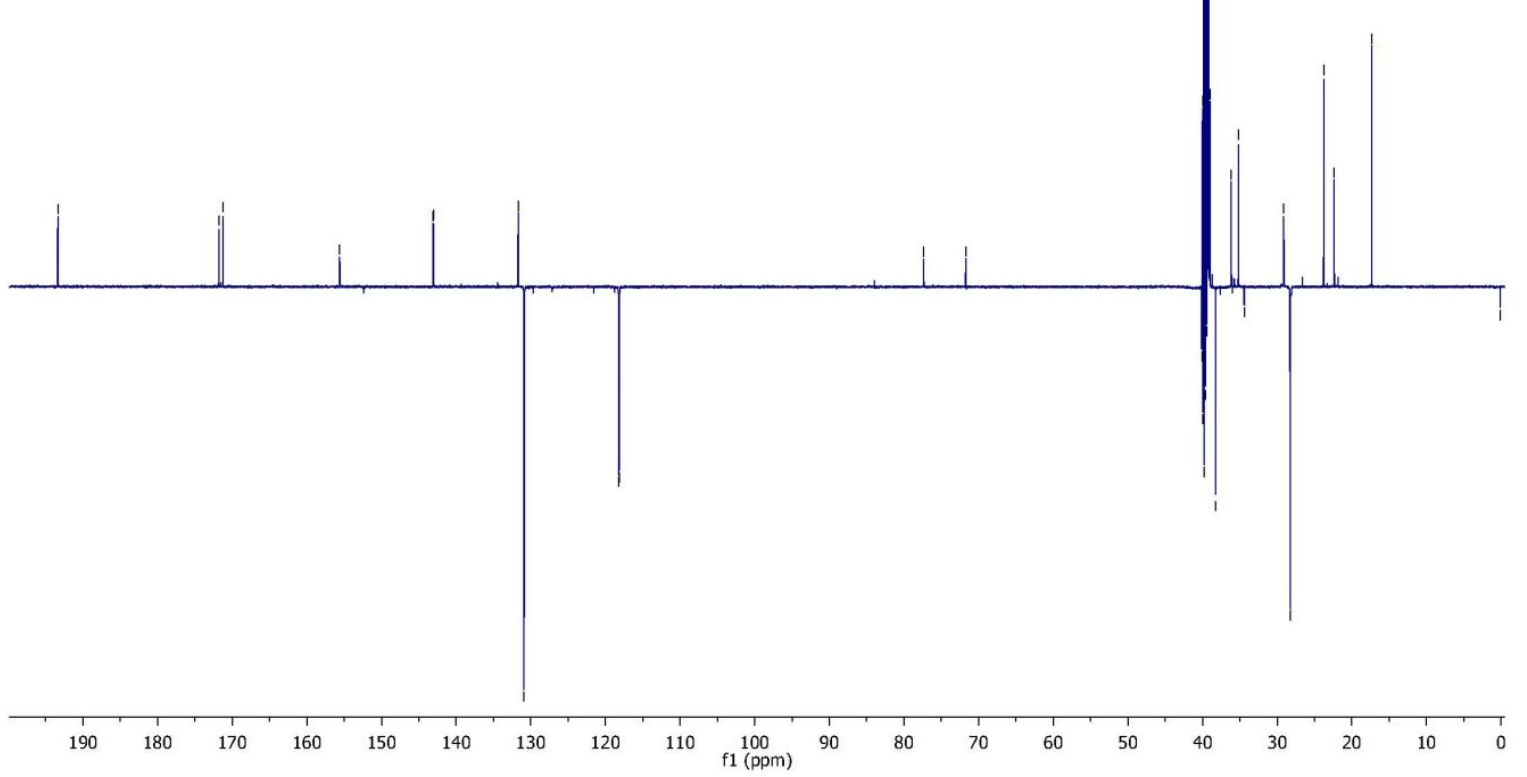


Graph SI-1. Inhibition percentages of various DNMT, KHMTs and PRMTs with $10 \mu \mathrm{M}$ of compound $\mathbf{1}, \mathbf{2}$ or $\mathbf{4}$

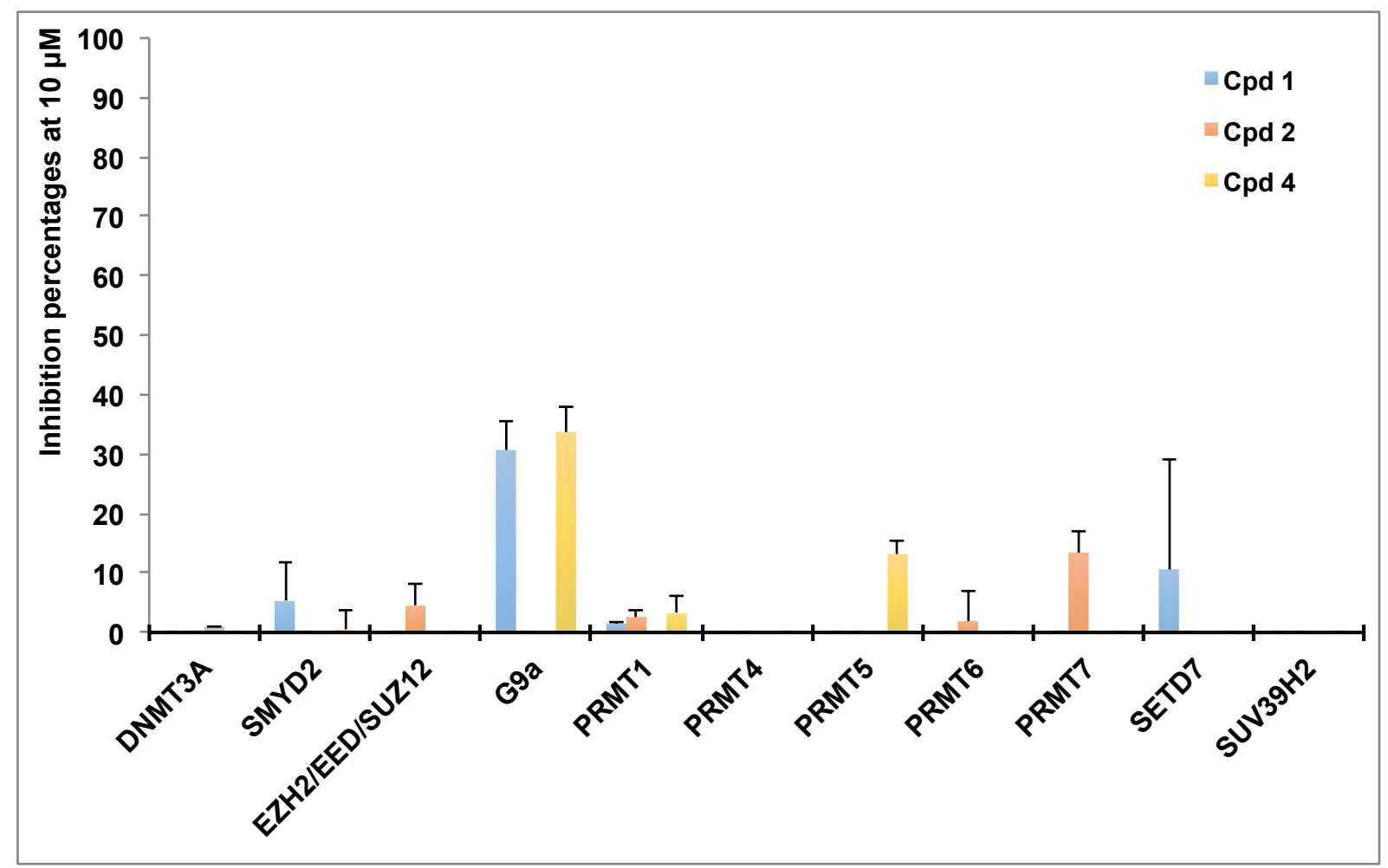


Table SI-1. Table of the 24 selected relevant proteins with significant enrichment profile after ABPP pull-down experiments with chemical probe 4 in KG-1 cell line versus control probe 5. Ratio and Student's T-test difference data are the means of the triplicates.

\begin{tabular}{|c|c|c|c|c|c|c|c|c|c|c|c|c|c|c|c|c|}
\hline Gene & Human Protein ID & $\begin{array}{c}\text { Mol. weight } \\
\text { (kDa) }\end{array}$ & \begin{tabular}{|c|} 
Student's T-test \\
Difference Probe \\
$\mathbf{4}$ in KG-1_Boc \\
Probe 5
\end{tabular} & $\begin{array}{c}\text { Probe } 4 \text { in } \mathrm{KG}- \\
1 / \text { Boc Probe } 5 \\
\text { ratio }\end{array}$ & \begin{tabular}{|c|} 
MS/MS \\
count Boc- \\
probe 5_1
\end{tabular} & \begin{tabular}{|c|} 
MS/MS \\
count Boc-- \\
probe 5_2
\end{tabular} & \begin{tabular}{|c|} 
KG-1 cell lin \\
MS/MS \\
count Boc- \\
probe $5{ }^{3} 3$
\end{tabular} & \begin{tabular}{|c|} 
MS/MS \\
count Probe \\
4_KG-1_1 \\
\end{tabular} & \begin{tabular}{|c|} 
MS/MS \\
count Probe \\
4_KG-1_2 \\
\end{tabular} & $\begin{array}{c}\text { MS/MS } \\
\text { count Probe } \\
\text { 4_KG-1_3 }\end{array}$ & \begin{tabular}{|c|} 
LFQ \\
intensity \\
Boc-probe \\
$5 \_1$ \\
\end{tabular} & \begin{tabular}{|c|} 
LFQ \\
intensity \\
Boc-probe \\
$5 \_2$ \\
\end{tabular} & \begin{tabular}{|c|} 
LFQ \\
intensity \\
Boc-probe \\
5 _3 \\
\end{tabular} & \begin{tabular}{|c|} 
LFQ \\
intensity \\
Probe 4_KG \\
$1 \_1$ \\
\end{tabular} & \begin{tabular}{|c|} 
LFQ \\
intensity \\
Probe 4_KG \\
$1 \_2$ \\
\end{tabular} & $\begin{array}{c}\text { LFQ } \\
\text { intensity } \\
\text { Probe 4_KG- } \\
1 \_3\end{array}$ \\
\hline SLC16A1 & P53985|MOT1 & 53.944 & 8.32 & 318.91 & 0 & 0 & 0 & 8 & 1 & 5 & \begin{tabular}{|l|l|}
$S 1$ & 1 \\
11.77 &
\end{tabular} & \begin{tabular}{|c|}
3.2 \\
13.05 \\
\end{tabular} & \begin{tabular}{|l|l|}
516.06 \\
16
\end{tabular} & \begin{tabular}{|l|l|}
$1 \leq 1$ \\
21.92 \\
\end{tabular} & \begin{tabular}{|l|l|}
12 & 2 \\
20.88 \\
\end{tabular} & 23.04 \\
\hline HSD17B4 & P51659|DHB4 & 79.685 & 7.17 & 143.56 & 0 & 2 & 0 & 26 & 9 & 19 & 14.55 & 16.71 & 15.53 & 23.47 & 21.07 & 23.74 \\
\hline SLC29A1 & Q99808|S29A1 & 50.219 & 6.51 & 90.97 & 0 & 0 & 0 & 5 & 1 & 6 & 14.14 & 14.74 & 13.53 & 21.35 & 18.89 & 21.67 \\
\hline HADHA & P40939|ECHA & 82.999 & 6.09 & 68.22 & 1 & 1 & 0 & 36 & 12 & 27 & 17.17 & 19.27 & 15.32 & 24.45 & 21.24 & 24.36 \\
\hline HSP9OB1 & P14625|ENPL & 92.468 & 5.34 & 40.61 & 9 & 10 & 2 & 84 & 244 & 73 & 22.77 & 23.30 & 21.95 & 28.80 & 26.50 & 28.75 \\
\hline KDSR & Q06136|KDSR & 36.187 & 4.63 & 24.79 & 0 & 0 & 0 & 5 & 2 & 1 & 13.14 & 14.18 & 14.10 & 19.59 & 18.05 & 17.68 \\
\hline HSD17B10 & Q99714|HCD2 & 26.923 & 4.02 & 16.19 & 0 & 1 & 0 & 1 & 4 & 2 & 12.78 & 16.60 & 12.95 & 17.77 & 18.94 & 17.67 \\
\hline SLC2A3 & P11169|GTR3;Q8TDB8|GTR14 & 53.924 & 4.00 & 16.01 & 0 & 0 & 0 & 4 & 2 & 2 & 15.94 & 15.36 & 15.26 & 19.45 & 19.62 & 19.49 \\
\hline NNT & Q13423|NNTM & 113.89 & 3.37 & 10.36 & 1 & 1 & 1 & 14 & 1 & 13 & 18.71 & 18.71 & 18.95 & 22.59 & 21.47 & 22.43 \\
\hline HM13 & Q8ТСТ9|нМ13 & 41.488 & 3.16 & 8.91 & 0 & 0 & 0 & 5 & 6 & 1 & 15.89 & 15.58 & 15.63 & 18.75 & 19.85 & 17.97 \\
\hline ATP5C1 & P36542|ATPG & 32.996 & 3.13 & 8.74 & 0 & 0 & 0 & 6 & 0 & 0 & 19.13 & 18.11 & 17.41 & 22.19 & 20.74 & 21.11 \\
\hline SLC16A3 & O15427|МОT4 & 49.469 & 2.97 & 7.83 & 0 & 0 & 0 & 4 & 1 & 4 & 16.66 & 17.02 & 17.00 & 19.54 & 19.84 & 20.21 \\
\hline NSDHL & Q15738|NSDHL & 41.9 & 2.76 & 6.76 & 0 & 0 & 0 & 4 & 0 & 4 & 15.63 & 16.07 & 15.83 & 18.59 & 18.73 & 18.47 \\
\hline PMPCA & Q10713|MPPA & 58.252 & 2.71 & 6.54 & 0 & 0 & 0 & 4 & 2 & 3 & 16.09 & 15.95 & 14.70 & 18.04 & 18.76 & 18.06 \\
\hline CPTIA & $\begin{array}{l}\text { P50416|CPT1A } \\
\text { P5T }\end{array}$ & 88.367 & 2.68 & 6.41 & 1 & 1 & 1 & 13 & 3 & 12 & 16.93 & 16.84 & 17.08 & 19.88 & 18.89 & 20.12 \\
\hline STाЗА & P46977|STा3A & 80.529 & 2.37 & 5.16 & 0 & 0 & 0 & 3 & 1 & 2 & 17.92 & 18.19 & 18.25 & 20.42 & 19.44 & 21.60 \\
\hline HSPD1 & P10809|CH60 & 61.054 & 2.34 & 5.06 & 29 & 37 & 9 & 60 & 145 & 58 & 24.74 & 24. & 23.77 & 26.86 & 26. & 26.98 \\
\hline LEPROTL1 & O95214|LERL1 & 14.428 & 2.28 & 4.86 & 0 & 0 & 0 & 0 & 3 & 3 & 11.57 & 11.39 & 11.46 & 12.21 & 14. & 14.51 \\
\hline SHMT2 & 397|GLYM & 55.992 & 1.68 & 3.21 & 2 & 3 & 0 & 8 & 4 & 6 & 19.96 & 20.35 & 19.09 & 21.36 & 22. & 21.03 \\
\hline SDHA & P31040|SDHA & 72.691 & 1.52 & 2.87 & 2 & 2 & 0 & 7 & 1 & 2 & 19.25 & 18.84 & 18.31 & 20.41 & 20.56 & 19.99 \\
\hline MRPL34 & Q9BQ48|RM34 & 10.165 & 1.45 & 2.73 & 0 & 0 & 0 & 3 & 1 & 2 & 15.04 & 15.47 & 15.44 & 16.42 & 17.37 & 16.51 \\
\hline MBOAT7 & Q96N66|MBOA7 & 52.764 & 1.36 & 2.56 & 1 & 1 & 0 & 2 & 3 & 3 & 16.64 & 16.69 & 16.47 & 17.86 & 18.34 & 17.67 \\
\hline SEC61A1 & P61619|S61A1 & & 1.1 & 2.2 & 3 & 2 & 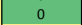 & 2 & 5 & 3 & 19.24 & 19.26 & 18.96 & 20.11 & 20.71 & 20.07 \\
\hline SF3A3 & $0.12874 \mid$ SF3A3 & 58.848 & 1.07 & 2.10 & 1 & 2 & 1 & 1 & 1 & 2 & 15.45 & 16.16 & 15.34 & 16.58 & 17.24 & 16.33 \\
\hline
\end{tabular}


Graph SI-2. Volcano plot of the proteomic data obtained following pull-down ABPP experiments with active chemical probe $\mathbf{4}$ in MCF-7 cell line and inactive probe $\mathbf{5}$.

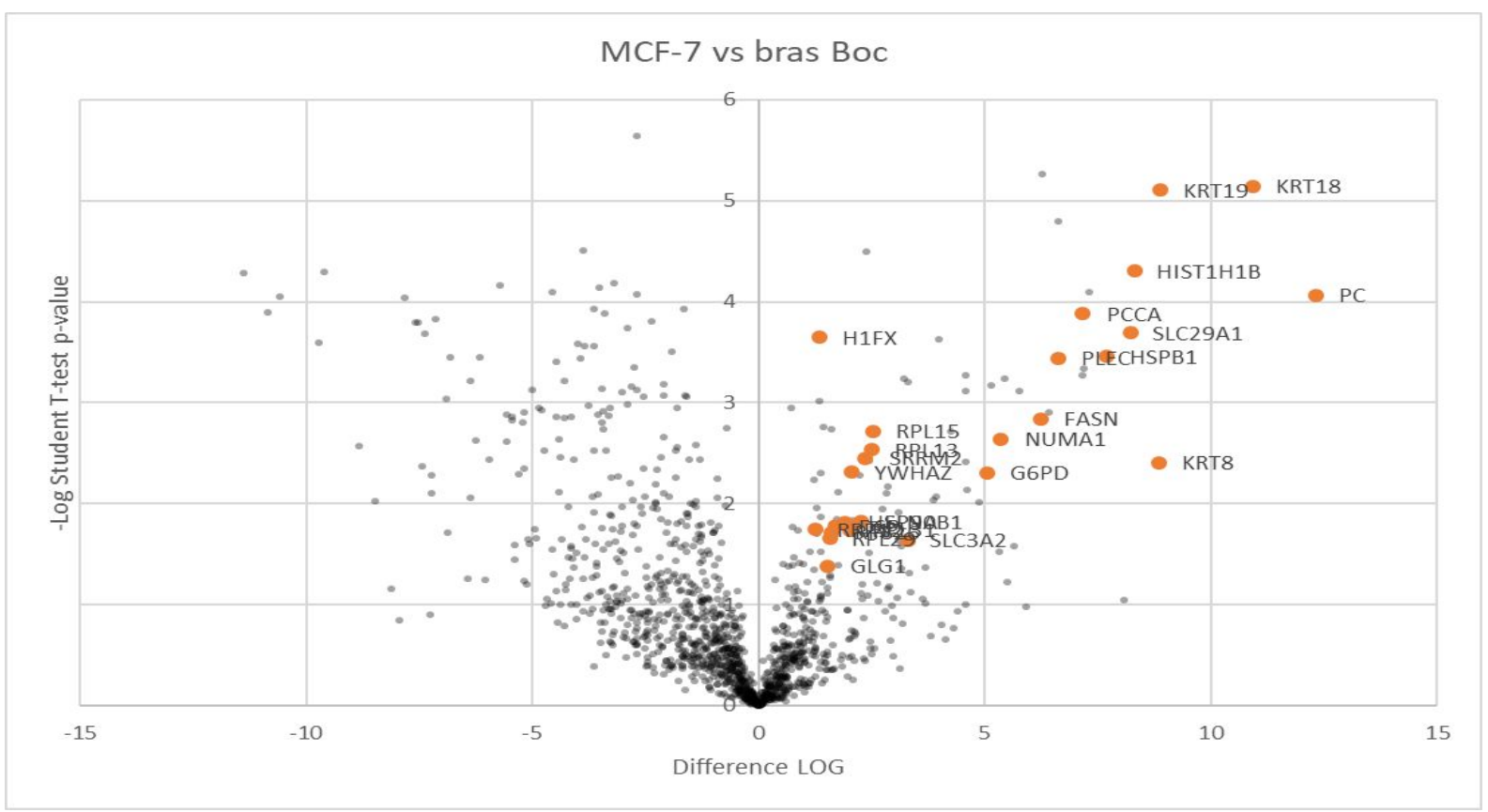


Table SI-2. Table of the 26 selected relevant proteins with significant enrichment profile after ABPP pull-down experiments with chemical probe 4 in MCF-7 cell line versus control probe 5. Ratio and Student's T-test difference data are the means of the triplicates.

\begin{tabular}{|c|c|c|c|c|c|c|c|c|c|c|c|c|c|c|c|c|}
\hline Gene & Human Protein ID & $\begin{array}{c}\text { Mol. weight } \\
(\mathrm{KDa})\end{array}$ & \begin{tabular}{|c|} 
Student's T-test \\
Difference Probe \\
4 in MCF-7_Boc \\
Probe 5
\end{tabular} & $\begin{array}{c}\text { Probe } \mathbf{4} \text { in } \\
\text { MCF- } 7 / \text { / Boc } \\
\text { Probe } 5 \text { ratio }\end{array}$ & \begin{tabular}{|c} 
MS/MS \\
count Boc- \\
probe 5_1
\end{tabular} & \begin{tabular}{|c|} 
MS/MS \\
count Boc- \\
probe 5_2 \\
\end{tabular} & \begin{tabular}{|c|}
$\begin{array}{c}\text { MSF-7 cell lins } \\
\text { count Boc- } \\
\text { probe 5_3 }\end{array}$ \\
\end{tabular} & \begin{tabular}{|c|} 
MS/MS \\
count Probe \\
4_MCF-7_1 \\
\end{tabular} & \begin{tabular}{|c|} 
MS/MS \\
count Probe \\
4_MCF-7_2
\end{tabular} & \begin{tabular}{|c|} 
MS/MS \\
count Probe \\
4_MCF-7_3
\end{tabular} & \begin{tabular}{|c|} 
LFQ \\
intensity \\
Boc-probe \\
5_1 \\
\end{tabular} & \begin{tabular}{|c|} 
LFQ \\
intensity \\
Boc-probe \\
$5 \_2$ \\
\end{tabular} & \begin{tabular}{|c|} 
LFQ \\
intensity \\
Boc-probe \\
$5-3$ \\
\end{tabular} & \begin{tabular}{|c|} 
LFQ \\
intensity \\
Probe \\
4_MCF-7_1 \\
\end{tabular} & \begin{tabular}{|c|} 
LFQ \\
intensity \\
Probe \\
4_MCF-7_2 2 \\
\end{tabular} & \begin{tabular}{|c} 
LFQ \\
intensity \\
Probe \\
4_MCF-7_3 \\
\end{tabular} \\
\hline $\mathrm{PC}$ & P11498|PYC & 129.63 & 12.33 & 5162.48 & 0 & 0 & 0 & 54 & 65 & 64 & 15.82 & 16.37 & 13.98 & 27.51 & 28.21 & 27.44 \\
\hline KRT18 & P05783|K1C18 & 48.057 & 10.92 & 1940.25 & 0 & 0 & 0 & 2 & 4 & 9 & 12.89 & 12.71 & 12.25 & 23.05 & 23.45 & 24.11 \\
\hline KRT19 & P08727|K1C19;CON_P08727 & 44.105 & 8.89 & 474.75 & 0 & 0 & 0 & 4 & 9 & 12 & 13.94 & 14.07 & 13.30 & 22.30 & 22.84 & 22.84 \\
\hline KRT8 & P05787|K2C8;CON_P05787 & 53.704 & 8.84 & 458.88 & 0 & 0 & 0 & 12 & 17 & 23 & 18.86 & 15.42 & 13.93 & 24.49 & 25.05 & 25.19 \\
\hline HIST1H1B & P16401|H15 & 22.58 & 8.32 & 320.56 & 0 & 0 & 0 & 4 & 1 & 1 & 13.28 & 13.50 & 12.43 & 21.75 & 21.65 & 20.78 \\
\hline SLC29A1 & Q99808|S29A1 & 50.219 & 8.24 & 301.73 & 0 & 0 & 0 & 2 & 3 & 9 & 14.14 & 14.74 & 13.53 & 21.46 & 22.35 & 23.30 \\
\hline HSPB1 & P04792 |HSPB1 & 22.782 & 7.71 & 208.93 & 0 & 0 & 0 & 2 & 3 & 4 & 14.85 & 15.87 & 13.55 & 22.28 & 22.64 & 22.46 \\
\hline PCCA & P05165|PCCA & 80.058 & 7.17 & 144.15 & 0 & 0 & 0 & 22 & 33 & 26 & 19.14 & 18.71 & 19.29 & 25.97 & 27.12 & 25.58 \\
\hline PLEC & Q15149|PLEC & 531.78 & 6.62 & 98.40 & 0 & 0 & 0 & 0 & 0 & 6 & 12.45 & 11.77 & 12.30 & 18.06 & 18.45 & 19.88 \\
\hline FASN & P49327|FAS & 273.42 & 6.23 & 75.00 & 0 & 0 & 0 & 1 & 8 & 8 & 16.50 & 17.00 & 15.07 & 21.34 & 23.14 & 22.76 \\
\hline NUMA1 & Q14980|NUMA1 & 238.26 & 5.37 & 41.23 & 0 & 0 & 0 & 3 & 1 & 3 & 13.13 & 15.25 & 13.10 & 19.11 & 18.71 & 19.76 \\
\hline GGPD & P11413|G6PD & 59.256 & 5.06 & 33.34 & 0 & 0 & 0 & 0 & 1 & 5 & 12.90 & 15.65 & 13.99 & 18.61 & 19.08 & 20.03 \\
\hline SLC3A2 & $=2$ & 93 & 3.30 & 9.82 & 2 & 1 & 0 & 2 & 1 & 1 & .03 & 16.36 & 13.62 & 18.52 & 18.16 & 19.21 \\
\hline RPL15 & P61313|RL15 & 24.146 & 2.54 & 5.80 & 9 & 7 & 7 & 9 & 13 & 14 & 23.50 & 23.30 & 24.42 & 26.28 & 26.19 & 26.35 \\
\hline RPL13 & P26373|RL13 & 261 & 2.52 & 5.73 & 2 & 2 & 3 & 7 & 6 & 11 & 22.54 & 22.63 & 23.46 & 25.86 & 24.97 & 25.36 \\
\hline SRRM2 & Q9UQ35|SRRM2 & 299.61 & 2.37 & 5.17 & 21 & 12 & 16 & 21 & 21 & 27 & 22.97 & 22.31 & 23.59 & 25.29 & 25.16 & 25.52 \\
\hline FLNA & P21333|FLNA & 280.74 & 2.27 & 4.81 & 2 & 4 & 1 & 2 & 3 & 8 & 19.17 & 19.40 & 18.79 & 20.48 & 21.38 & 22.31 \\
\hline YWHAZ & P63104|14332 & 27.745 & 2.06 & 4.18 & 3 & 4 & 4 & 4 & 3 & 6 & 20.34 & 20.45 & 19.72 & 21.68 & 22.37 & 22.64 \\
\hline RPL31 & $\begin{array}{l}\text { P62899|RLL31 } \\
\end{array}$ & 14.463 & 2.02 & 4.05 & 4 & 5 & 10 & 3 & 5 & 5 & 21.67 & 21.83 & 22.55 & 24.57 & 24.40 & 23.14 \\
\hline HSP9OB1 & $\begin{array}{l}\text { P14625|ENPL } \\
\text { P14 }\end{array}$ & 92.468 & 1.92 & 3.78 & 9 & 10 & 2 & 6 & 8 & 22 & 22.77 & 23.30 & 21.95 & 24.12 & 24.62 & 25.04 \\
\hline DSP & P15924|DESP & 331.77 & 1.69 & 3.23 & 5 & 1 & 2 & 1 & 1 & 3 & 17.58 & 16.47 & 17.41 & 18.70 & 18.50 & 19.34 \\
\hline RPS26 & |P62854|RS26;Q5JNZ5|RS26L & 13.015 & 1.61 & 3.06 & 1 & 3 & 1 & 3 & 3 & 2 & 18.48 & 18.76 & 19.25 & 21.07 & 20.44 & 19.82 \\
\hline RPL29 & P47914|RL29 & 17.752 & 1.57 & 2.98 & 4 & 2 & 1 & 1 & 1 & 1 & 21.79 & 21.38 & 22.74 & 23.83 & 23.33 & 23.46 \\
\hline GLG1 & Q92896|GSLG1 & 134.55 & 1.53 & 2.89 & 2 & 1 & 1 & 0 & 1 & 1 & 13.90 & 12.93 & 14.09 & 14.84 & 14.76 & 15.91 \\
\hline $\mathrm{H} 1 \mathrm{FX}$ & Q92522|H1X & 22.487 & 1.33 & 2.52 & 1 & 1 & 1 & 1 & 1 & 1 & 18.69 & 19.01 & 18.74 & 20.19 & 20.09 & 20.17 \\
\hline RRP12 & Q5JTH9|RRP12 & 143.7 & 1.24 & 2.37 & 2 & 2 & 3 & 0 & 0 & 1 & 16.90 & 17.08 & 17.88 & 18.59 & 18.32 & 18.68 \\
\hline
\end{tabular}


Graph SI-3. Volcano plot of the proteomic data obtained following pull-down ABPP experiments with active chemical probe 4 in K-562 cell line and inactive probe 5 .

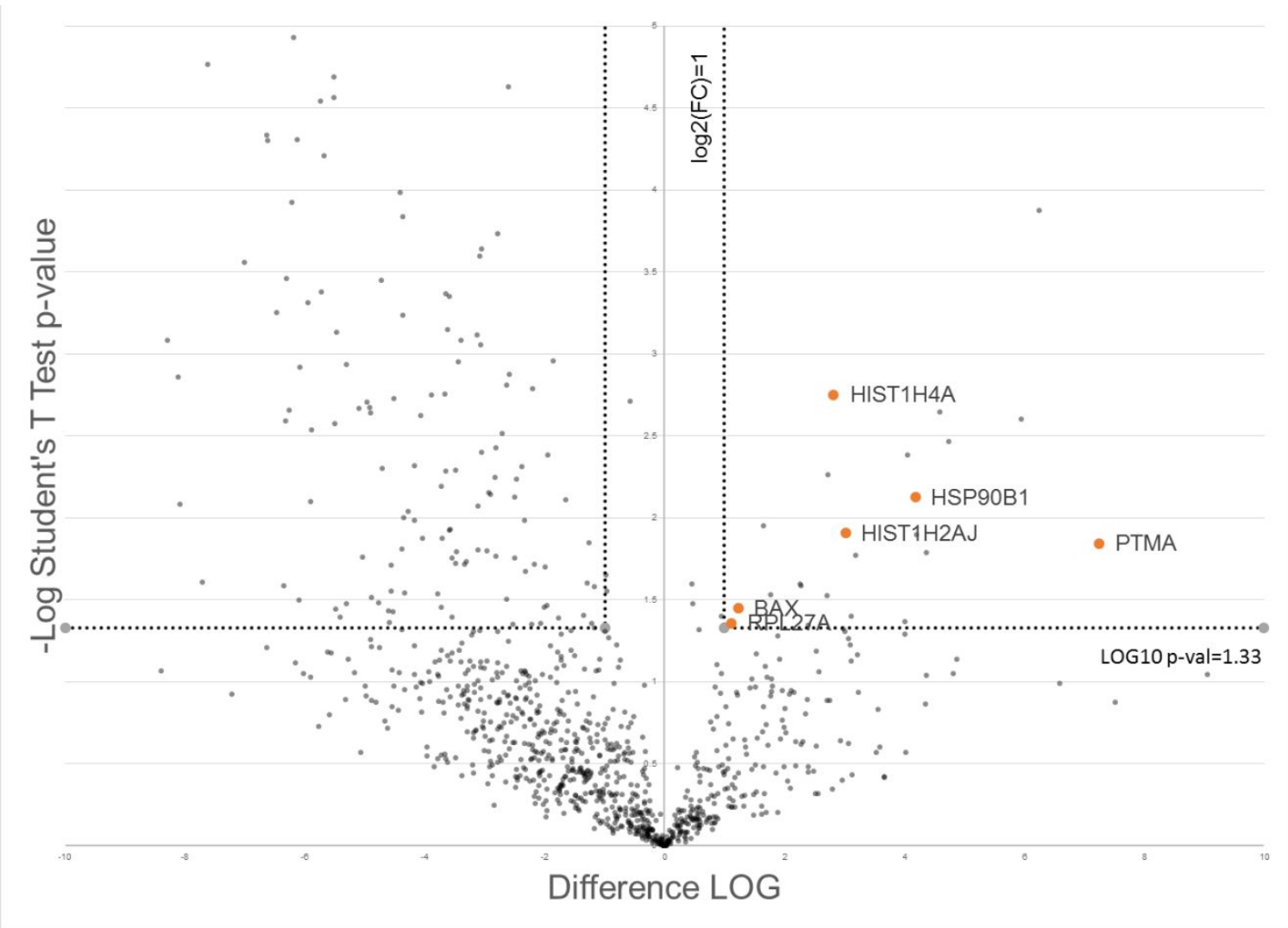


Table SI-3. Table of the 6 selected relevant proteins with significant enrichment profile after ABPP pull-down experiments with chemical probe 4 in K-562 cell line versus control probe 5. Ratio and Student's T-test difference data are the means of the triplicates.

\begin{tabular}{|c|c|c|c|c|c|c|c|c|c|c|c|c|c|c|c|c|}
\hline Gene & Human Protein ID & $\begin{array}{l}\text { Mol. weight } \\
\text { (kDa) }\end{array}$ & \begin{tabular}{|c|} 
Student's T-test \\
Difference Probe \\
4 in K-562_Boc \\
Probe 5
\end{tabular} & \begin{tabular}{|c} 
Probe 4 in K- \\
$562 /$ Boc \\
Probe 5 ratio
\end{tabular} & \begin{tabular}{|c|} 
MS/MS \\
count Boc- \\
probe 5_1
\end{tabular} & \begin{tabular}{|c} 
MS/MS \\
count Boc- \\
probe 5_2
\end{tabular} & $\begin{array}{c}\text { MS/MS } \\
\text { count Boc- } \\
\text { probe 5_3 }\end{array}$ & \begin{tabular}{|c|} 
MS/MS \\
count Probe \\
4_K-562_1
\end{tabular} & \begin{tabular}{|c|} 
MS/MS \\
count Probe \\
4_K- $562 \_2$ \\
\end{tabular} & $\begin{array}{c}\text { MS/MS } \\
\text { count Probe } \\
\text { 4_-5-562_3 }\end{array}$ & \begin{tabular}{|c|} 
LFQ \\
intensity \\
Boc-probe \\
$5 \_1$
\end{tabular} & \begin{tabular}{|c|} 
LFQ \\
intensity \\
Boc-probe \\
$5 \_2$ \\
\end{tabular} & \begin{tabular}{|c|} 
LFQ \\
intensity \\
Boc-probe \\
$5 \_3$ \\
\end{tabular} & \begin{tabular}{|c} 
LFQ \\
intensity \\
Probe 4_K- \\
$562 \_1$
\end{tabular} & \begin{tabular}{|c|} 
LFO \\
intensity \\
Probe 4_K- \\
562 _2
\end{tabular} & \begin{tabular}{|c|} 
LFQ \\
intensity \\
Probe 4_K- \\
$562 \_3$ \\
. 3
\end{tabular} \\
\hline $\begin{array}{l}\text { PTMA } \\
\end{array}$ & $\begin{array}{l}\text { P06454|PTMA } \\
\end{array}$ & 12.203 & 7.25 & 152.49 & 0 & 0 & 0 & 0 & 25 & 0 & 12.93 & 12.76 & 12.36 & 17.83 & 23.39 & 18.59 \\
\hline HSP90B1 & P14625|ENPL & 92.468 & 4.19 & 18.22 & 9 & 10 & 2 & 34 & 19 & 25 & 22.77 & 23.30 & 21.95 & 28.34 & 26.20 & 26.04 \\
\hline BAX & Q07812|BAX & 21.184 & 1.23 & 2.34 & 3 & 4 & 2 & 2 & 0 & 2 & 23.25 & 23.73 & 23.16 & 26.67 & 27.40 & 25.11 \\
\hline RPL27A & P46776|RL27A & 16.561 & 1.12 & 2.17 & 0 & 1 & 1 & 1 & 1 & 5 & 24.71 & 25.01 & 25.90 & 28.13 & 28.15 & 27.77 \\
\hline HIST1H2AJ & Q999878|Н2A;Q96KК5|Н2А1Н; & d 13.936 & 3.02 & 8.09 & 4 & 4 & 3 & 5 & 5 & 6 & 16.98 & 16.65 & 17.57 & 18.35 & 17.77 & 18.76 \\
\hline HIST1H4A & P62805|H4 & 11.367 & 2.81 & 7.02 & 6 & 8 & 9 & 16 & 8 & 39 & 20.84 & 21.22 & 22.01 & 22.52 & 22.76 & 22.15 \\
\hline
\end{tabular}


Graph SI-4. Volcano plot of the proteomic data obtained following pull-down ABPP experiments with active chemical probe 4 in MOLM-13 cell line and inactive probe $\mathbf{5}$.

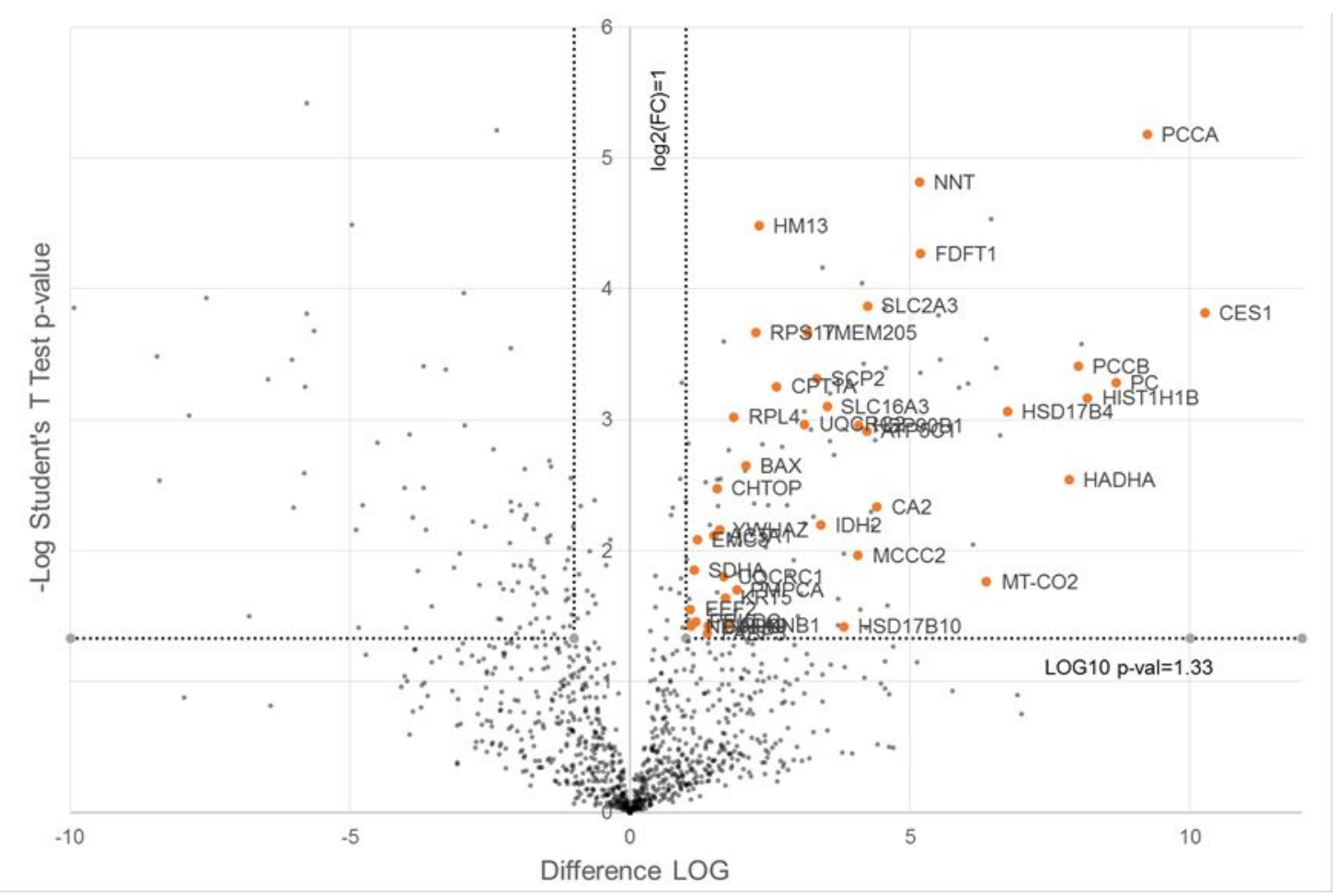


Table SI-4. Table of the 40 selected relevant proteins with significant enrichment profile after ABPP pull-down experiments with chemical probe $\mathbf{4}$ in MOLM-13 cell line versus control probe 5. Ratio and Student's T-test difference data are the means of the triplicates.

\begin{tabular}{|c|c|c|c|c|c|c|c|c|c|c|c|c|c|c|c|c|}
\hline Gene & Human Protein ID & $\begin{array}{l}\text { Mol. weight } \\
\text { (kDa) }\end{array}$ & \begin{tabular}{|c|} 
Student's T-test \\
Difference Probe \\
4 in MOLM- \\
13 Boc Probe 5 \\
\end{tabular} & $\begin{array}{c}\text { Probe } 4 \text { in } \\
\text { MOLM- } \\
13 / \text { Boc Probe } \\
5 \text { ratio }\end{array}$ & $\begin{array}{c}\text { MS/MS } \\
\text { count Boc- } \\
\text { probe 5_1 }\end{array}$ & \begin{tabular}{|c|} 
MS/MS \\
count Boc- \\
probe 5_2
\end{tabular} & \begin{tabular}{|c|} 
MS/MS \\
count Boc- \\
probe 5_3 \\
\end{tabular} & \begin{tabular}{|c|} 
Iine \\
MS/MS \\
count Probe \\
4_MoLM- \\
$13 \_1$ \\
\end{tabular} & \begin{tabular}{|c|} 
MS/MS \\
count Probe \\
4_MLLM- \\
$13 \_22$ \\
\end{tabular} & \begin{tabular}{|c|} 
MS/MS \\
count Probe \\
4_MLLM- \\
$13 \_3$ \\
\end{tabular} & $\begin{array}{c}\text { LFQ } \\
\text { intensity } \\
\text { Boc-probe } \\
\mathbf{5} \text { _1 }\end{array}$ & \begin{tabular}{|c|} 
LFQ \\
intensity \\
Boc-probe \\
$5 \_2$ \\
\end{tabular} & \begin{tabular}{|c|} 
LFQ \\
intensity \\
Boc-probe \\
$5{ }_{-} 3$ \\
\end{tabular} & \begin{tabular}{|c|} 
LFQ \\
intensity \\
Probe \\
4_MOLM- \\
13__1 \\
\end{tabular} & $\begin{array}{c}\text { LFQ } \\
\text { intensity } \\
\text { Probe } \\
\text { 4_MOLM- } \\
\text { 13_2 } \\
\end{array}$ & $\begin{array}{c}\text { LFQ } \\
\text { intensity } \\
\text { Probe } \\
\text { 4_MOLM- } \\
\text { 13_3 }\end{array}$ \\
\hline CES1 & P23141|EST1 & 62.52 & 10.27 & 1230.50 & 0 & 0 & 0 & 6 & 3 & 4 & 12.50 & 10.88 & 13.20 & 22.67 & 21.94 & 22.76 \\
\hline PCCA & P05165|PCCA & 80.058 & 9.23 & 600.93 & 0 & 0 & 0 & 53 & 55 & 48 & 19.14 & 18.71 & 19.29 & 27.80 & 28.49 & 28.56 \\
\hline & P11498|PYC & 129.63 & 8.67 & 407.86 & 0 & 0 & 0 & 33 & 25 & 31 & 15.82 & 16.37 & 13.98 & 23.16 & 24.55 & 24.46 \\
\hline HIST1H1B & P16401|H15 & 22.58 & 8.17 & 287.34 & 0 & 0 & 0 & 11 & 4 & 1 & 13.28 & 13.50 & 12.43 & 22.70 & 21.03 & 19.97 \\
\hline PCCB & P05166|PCCB & 58.215 & 8.00 & 256.39 & 0 & 0 & 0 & 5 & 6 & 4 & 13.58 & 11.43 & 11.70 & 19.69 & 20.57 & 20.46 \\
\hline HADHA & P40939|ECHA & 82.999 & 8.84 & 228.70 & 1 & 1 & 0 & 33 & 22 & 25 & 17.17 & 19.27 & 15.32 & 25.55 & 24.33 & 25.41 \\
\hline HSD17B4 & P51659|DHB4 & 79.685 & 6.74 & 106.86 & 0 & 2 & 0 & 22 & 7 & 8 & 14.55 & 16.71 & 15.53 & 22.90 & 21.51 & 22.59 \\
\hline MT-CO2 & P00403|COX2 & 25.565 & 6.36 & 82.23 & 0 & 0 & 0 & 3 & 2 & 1 & 14.63 & 18.46 & 13.03 & 22.13 & 21.55 & 21.52 \\
\hline FDFT1 & P37268|FDFT & 48.115 & 5.19 & 36.46 & 0 & 0 & 0 & 3 & 1 & 2 & 12.82 & 12.32 & 13.19 & 17.89 & 17.77 & 18.22 \\
\hline NNT & Q13423|NNTM & 113.89 & 5.16 & 35.77 & 1 & 1 & 1 & 26 & 11 & 15 & 18.71 & 18.71 & 18.95 & 24.21 & 23.58 & 24.06 \\
\hline CA2 & P00918|CAH2 & 29.246 & 4.41 & 21.26 & 0 & 0 & 0 & 4 & 1 & 1 & 13.14 & 11.42 & 13.62 & 17.54 & 17.51 & 16.36 \\
\hline SLC2A3 & P11169|GTR3;Q8TDB8|GTR14 & 53.924 & 4.24 & 18.85 & 0 & 0 & 0 & 4 & 5 & 4 & 15.94 & 15.36 & 15.26 & 19.80 & 19.38 & 20.09 \\
\hline ATPSC1 & P36542|ATPG & 32.996 & 4.22 & 18.68 & 0 & 0 & 0 & 3 & 4 & 4 & 19.13 & 18.11 & 17.41 & 22.33 & 22.29 & 22.70 \\
\hline HSP9OB1 & P14625|ENPL & 92.468 & 4.08 & 16.87 & 9 & 10 & 2 & 41 & 30 & 33 & 22.77 & 23.30 & 21.95 & 26.72 & 26.27 & 27.26 \\
\hline MCCC2 & Q9HCCO|MCCB & 61.332 & 4.07 & 16.79 & 0 & 0 & 0 & 4 & 2 & 0 & 14.44 & 16.15 & 13.10 & 18.79 & 18.87 & 18.23 \\
\hline HSD17B10 & Q99714|HCD2 & 26.923 & 3.82 & 14.08 & 0 & 1 & 0 & 2 & 2 & 3 & 12.78 & 16.60 & 12.95 & 18.12 & 17.87 & 17.78 \\
\hline SLC16A3 & О15427|МOT4 & 49.469 & 3.52 & 11.44 & 0 & 0 & 0 & 3 & 2 & 2 & 16.66 & 17.02 & 17.00 & 21.05 & 19.78 & 20.39 \\
\hline IDH2 & P48735|IDHP & 50.909 & 3.41 & 10.60 & 0 & 0 & 0 & 3 & 5 & 3 & 16.61 & 14.80 & 14.58 & 18.64 & 18.94 & 18.64 \\
\hline SCP2 & P22307|NLTP & 58.993 & 3.33 & 10.03 & 2 & 1 & 1 & 4 & 4 & 4 & 18.04 & 18.27 & 18.47 & 22.08 & 21.05 & 21.64 \\
\hline TMEM205 & Q6UW68|TM205 & 21.198 & 3.18 & 9.04 & 1 & 0 & 0 & 3 & 1 & 3 & 18.10 & 17.50 & 17.95 & 21.37 & 20.89 & 20.82 \\
\hline UQCRC2 & P22695|QCR2 & 48.442 & 3.11 & 8.64 & 1 & 3 & 0 & 7 & 4 & 3 & 18.51 & 18.51 & 17.67 & 21.80 & 20.99 & 21.24 \\
\hline CPT1A & $\begin{array}{l}\text { P50416|CPT1A } \\
\text { I T }\end{array}$ & 88.367 & 2.62 & 6.13 & 1 & 1 & 1 & 5 & 8 & 9 & 16.93 & 16.84 & 17.08 & 19.36 & 19.27 & 20.07 \\
\hline HM13 & Q8TCT9|HM13 & 41.488 & 2.30 & 4.91 & 0 & 0 & 0 & 3 & 2 & 2 & 15.89 & 15.58 & 15.63 & 17.95 & 18.11 & 17.92 \\
\hline RPS17 & P08708|RS17 & 15.55 & 2.24 & 4.73 & 3 & 5 & 2 & 5 & 2 & 2 & 18.91 & 19.06 & 19.28 & 21.58 & 21.10 & 21.29 \\
\hline BAX & Q07812|BAX & $\begin{array}{l}11.133 \\
21.184\end{array}$ & $\begin{array}{l}2.24 \\
2.07\end{array}$ & 4.18 & 3 & 4 & 2 & 4 & 3 & 4 & $\begin{array}{l}18.91 \\
16.98\end{array}$ & $\begin{array}{l}19.60 \\
16.65\end{array}$ & $\begin{array}{l}19.28 \\
17.57\end{array}$ & 19.26 & 18.88 & 19.24 \\
\hline $\begin{array}{l}\text { PMPCA } \\
\text { PMPA }\end{array}$ & Q10713|MPPA & $\begin{array}{l}58.252 \\
58.104\end{array}$ & $\begin{array}{l}2.90 \\
1.90\end{array}$ & $\begin{array}{l}4.18 \\
3.74\end{array}$ & 0 & 0 & 0 & $\begin{array}{l}4 \\
3\end{array}$ & 1 & $\begin{array}{l}4 \\
2\end{array}$ & $\begin{array}{l}10.90 \\
16.09\end{array}$ & $\begin{array}{l}10.05 \\
15.95\end{array}$ & $\begin{array}{l}14.70 \\
\end{array}$ & 17.78 & $\begin{array}{l}10.08 \\
16.98\end{array}$ & $\begin{array}{l}19.24 \\
17.68\end{array}$ \\
\hline RPL4 & P36578|RL4 & $\begin{array}{l}47.697 \\
4.252\end{array}$ & 1.85 & $\begin{array}{l}3.14 \\
3.60\end{array}$ & 8 & 5 & 3 & 11 & 13 & 9 & 21.92 & 21.74 & 22.35 & 23.66 & $\begin{array}{l}24.03 \\
24.03\end{array}$ & $\begin{array}{l}23.08 \\
23.87\end{array}$ \\
\hline PLXNB1 & 043157|PLXB1 & 232.3 & 1.73 & 3.33 & 0 & 0 & 0 & 1 & 4 & 1 & 26.02 & 25.95 & 26.63 & 26.91 & 28.61 & 28.28 \\
\hline KRTS & P13647|K2C5;CON_P13647 & 62.378 & 1.7 & 3.2 & 0 & 0 & 0 & 1 & 4 & 1 & 20.45 & 19.97 & 20.59 & 21.26 & 22.12 & 22.76 \\
\hline UQCRC1 & P31930|QCR1 & 52.645 & 1.66 & 3.17 & 1 & 2 & 0 & 3 & 3 & 2 & 19.04 & 18.67 & 17.85 & 20.60 & 19.85 & 20.11 \\
\hline YWHAZ & P63104|143 & 27.745 & 1.6 & 3.0 & 3 & 4 & 4 & 7 & 6 & 6 & 20.34 & 20.45 & 19.72 & 22.17 & 21.68 & 21.44 \\
\hline CHTOP & Q9Y3Y2|CHTOP & 26.396 & 1.5 & 2.9 & 2 & 2 & 2 & 3 & 2 & 1 & 16.81 & 16.39 & 17.13 & 18.16 & 18.24 & 18.57 \\
\hline ACTA1 & P68133|ACTS;P68032|ACTC;P6: & 42.051 & 1.5 & $2.8=$ & 5 & 2 & 2 & 1 & 3 & 2 & 21.87 & 22.42 & 21.38 & 23.33 & 23.47 & 23.37 \\
\hline CAPG & P40121|CAPG & 38.498 & 1.3 & 2.63 & 5 & 4 & 1 & 3 & 4 & 3 & 18.64 & 18.86 & 17.54 & 20.10 & 19.72 & 19.40 \\
\hline FABP5 & & 15.164 & 1.3 & 2.6 & 1 & 1 & 2 & 5 & 2 & 0 & 17.34 & 17.57 & 17.81 & 19.79 & 18.84 & 18.23 \\
\hline EMC3 & Q9PO & 29.952 & 1.2. & 2.2 & 0 & 0 & 0 & 5 & 1 & 1 & 13.59 & 13.23 & 13.98 & 14.58 & 14.84 & 14.98 \\
\hline PRKDC & & 469.08 & 1.1. & 2.2 & 0 & 1 & 0 & 1 & 5 & 2 & 19.01 & 19.86 & 19.89 & 20.29 & 20.89 & 21.07 \\
\hline SDHA & P3 & 72.691 & 1.14 & 2.21 & 2 & 2 & 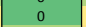 & 2 & 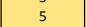 & 2 & 19.25 & 18.84 & 18.31 & 19.94 & 19.99 & 19.90 \\
\hline NDUFA9 & Q167 & 42.509 & 1.08 & 2.12 & 3 & 2 & 1 & 6 & 4 & 2 & 20.22 & 20.11 & 20.61 & 21.91 & 21.48 & 20.81 \\
\hline EEF2 & P13639|EF2 & 95.337 & $\begin{array}{l}1.07 \\
\end{array}$ & 2.11 & 7 & 8 & 6 & 13 & 11 & 5 & 21.12 & 21.35 & 20.37 & 21.98 & $\begin{array}{l}21.40 \\
22.24\end{array}$ & 21.84 \\
\hline
\end{tabular}


Graph SI-5. Volcano plot of the proteomic data obtained following pull-down ABPP experiments with active chemical probe 4 and inactive probe 5 in WM-266-4 cell line.

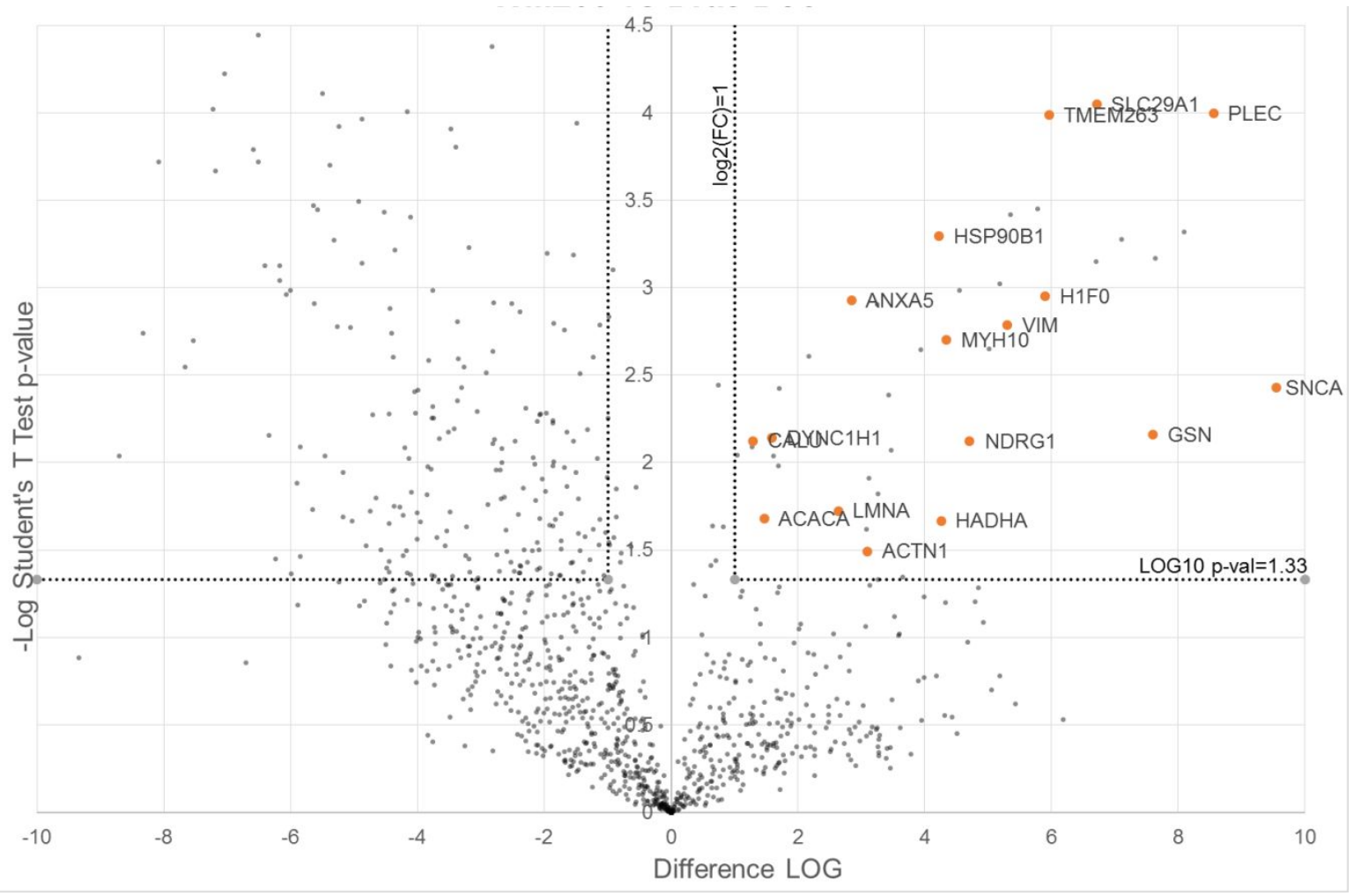


Table SI-5. Table of the 19 selected relevant proteins with significant enrichment profile after ABPP pull-down experiments with chemical probe 4 in WM-266-4 cell line versus control probe 5. Ratio and Student's T-test difference data are the means of the triplicates.

\begin{tabular}{|c|c|c|c|c|c|c|c|c|c|c|c|c|c|c|c|c|}
\hline Gene & Human Protein ID & $\begin{array}{c}\text { Mol. weight } \\
(\mathrm{KDa})\end{array}$ & \begin{tabular}{|c|} 
Student's T-test \\
Difference Probe \\
4 in WM-266- \\
4_Boc Probe 5 \\
\end{tabular} & $\begin{array}{c}\begin{array}{c}\text { Probe } 4 \text { in } \\
\text { WM-266- } \\
4 / \text { Boc Probe } 5 \\
\text { ratio }\end{array} \\
\end{array}$ & $\begin{array}{c}\text { MS/MS } \\
\text { count Boc- } \\
\text { probe 5_1 }\end{array}$ & $\begin{array}{c}\text { MS/MS } \\
\text { count Boc- } \\
\text { probe 5_2 }\end{array}$ & \begin{tabular}{|c|} 
MS/MS \\
count Boc- \\
probe 5_3 3
\end{tabular} & \begin{tabular}{|c|} 
MS/MS \\
count Probe \\
4-WM-266- \\
$4 \_1$
\end{tabular} & $\begin{array}{c}\text { MS/MS } \\
\text { count Probe } \\
4 \text { _WM-266- } \\
4 \_2\end{array}$ & \begin{tabular}{|c|} 
MS/MS \\
count Probe \\
4-WM-266- \\
4 4_3
\end{tabular} & $\begin{array}{c}\text { LFQ } \\
\text { intensity } \\
\text { Boc-probe } \\
\mathbf{5}{ }_{-1}\end{array}$ & $\begin{array}{c}\text { LFQ } \\
\text { intensity } \\
\text { Boc-probe } \\
5 \_2\end{array}$ & $\begin{array}{c}\text { LFQ } \\
\text { intensity } \\
\text { Boc-probe } \\
5 \_3\end{array}$ & \begin{tabular}{|c|} 
LFQ \\
intensity \\
Probe \\
4_WM-266- \\
4_1 \\
\end{tabular} & \begin{tabular}{|c|} 
LFQ \\
intensity \\
Probe \\
4_WM-266- \\
4_2 \\
\end{tabular} & $\begin{array}{c}\text { LFQ } \\
\text { intensity } \\
\text { Probe } \\
\text { 4_WM-266- } \\
\text { 4_-3 }\end{array}$ \\
\hline PC & P11498|PYC & 129.63 & 13.15 & 9116.51 & 0 & 0 & 0 & 97 & 97 & 72 & 15.82 & 16.37 & 13.98 & 28.78 & 28.70 & 28.14 \\
\hline SNCA & P37840|SYUA & 14.46 & 9.54 & 746.09 & 0 & 0 & 0 & 12 & 0 & 1 & 12.52 & 11.87 & 15.03 & 25.17 & 21.59 & 21.29 \\
\hline PCCA & P05165|PCCA & 80.058 & 9.15 & 568.88 & 0 & 0 & 0 & 45 & 54 & 46 & 19.14 & 18.71 & 19.29 & 28.93 & 27.88 & 27.80 \\
\hline PLEC & Q15149|PLEC & 531.78 & 8.56 & 378.07 & 0 & 0 & 0 & 15 & 8 & 9 & 12.45 & 11.77 & 12.30 & 21.19 & 21.30 & 19.71 \\
\hline GSN & P06396|GELS_HUMAN;CON_C & 85.696 & 7.61 & 194.85 & 0 & 0 & 0 & 5 & 2 & 15 & 15.63 & 15.04 & 11.50 & 21.66 & 20.39 & 22.95 \\
\hline SLC29A1 & Q99808|S29A1 & 50.279 & 6.72 & 105.60 & 0 & 0 & 0 & 2 & 5 & 3 & 14.14 & 14.74 & 13.53 & 21.15 & 21.02 & 20.39 \\
\hline TMEM263 & Q8WUH6|TM263 & 11.748 & 5.96 & 62.26 & 0 & 0 & 0 & 2 & 2 & 4 & 13.13 & 13.27 & 13.40 & 19.67 & 19.55 & 18.48 \\
\hline H1F0 & P07305|H10 & 20.863 & 5.90 & 59.58 & 0 & 0 & 0 & 2 & 3 & 1 & 12.36 & 12.17 & 14.02 & 17.97 & 19.08 & 19.19 \\
\hline VIM & P08670|VIME & 53.651 & 5.30 & 39.43 & 3 & 2 & 0 & 18 & 11 & 26 & 19.18 & 18.95 & 17.67 & 24.48 & 22.87 & 24.36 \\
\hline NDRG1 & Q92597|NDRG1 & 42.835 & 4.71 & 26.19 & 0 & 0 & 0 & 3 & 1 & 3 & 16.13 & 15.49 & 13.16 & 20.03 & 19.08 & 19.80 \\
\hline MYH10 & Р35580|MYH10 & 229 & 4.35 & 20.33 & 0 & 0 & 0 & 6 & 2 & 2 & 12.52 & 14.21 & 14.00 & 18.46 & 17.86 & 17.46 \\
\hline HADHA & P40939|ECHA & 82.999 & 4.27 & 19.25 & 1 & 1 & 0 & 3 & 2 & 4 & 17.17 & 19.27 & 15.32 & 21.17 & 21.42 & 21.98 \\
\hline HSP9OB1 & P14625|ENPL & 92.468 & 4.23 & 18.74 & 9 & 10 & 2 & 25 & 25 & 27 & 22.77 & 23.30 & 21.95 & 27.15 & 26.78 & 26.77 \\
\hline ACTN1 & P12814|ACTN1 & 103.06 & 3.09 & 8.52 & 0 & 1 & 0 & 2 & 0 & 4 & 13.78 & 16.50 & 16.51 & 19.18 & 18.11 & 18.76 \\
\hline ANXA5 & P08758|ANXA5 & 35.936 & 2.84 & 7.18 & 3 & 1 & 0 & 8 & 4 & 10 & 20.61 & 20.31 & 19.64 & 23.16 & 22.65 & 23.27 \\
\hline LMNA & P02545|LMNA & 74.139 & 2.64 & 6.22 & 0 & 3 & 0 & 8 & 2 & 12 & 19.04 & 19.75 & 18.55 & 21.79 & 20.69 & 22.77 \\
\hline DYNC1H1 & Q14204|DYHC1 & 532.4 & 1.59 & 3.02 & 0 & 2 & 0 & 5 & 1 & 1 & 17.24 & 17.44 & 17.92 & 19.59 & 19.02 & 18.77 \\
\hline ACACA & Q13085|ACACA & 265.55 & 1.47 & & 22 & 27 & 23 & & 34 & 34 & 24.29 & 24.38 & 25.28 & 26.32 & 26.39 & 25.63 \\
\hline CALU & O43852|CALU & 37.106 & 1.30 & 2.46 & 3 & 1 & 1 & 2 & 1 & 2 & 15.56 & 15.41 & 15.41 & 17.21 & 16.32 & 16.74 \\
\hline
\end{tabular}

